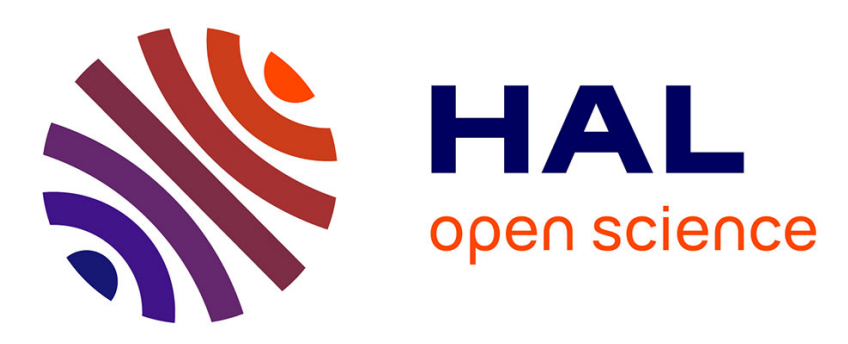

\title{
Fast and Accurate Multiplicative Decomposition for Fringe Removal in Interferometric Images
}

Daniel-Chen Soncco, Clara Barbanson, Mila Nikolova, Andrés Almansa, Yann Ferrec

\section{- To cite this version: \\ Daniel-Chen Soncco, Clara Barbanson, Mila Nikolova, Andrés Almansa, Yann Ferrec. Fast and Accu- rate Multiplicative Decomposition for Fringe Removal in Interferometric Images. IEEE Transactions on Computational Imaging, 2017, 3 (2), 10.1109/TCI.2017.2678279 . hal-01492428}

\section{HAL Id: hal-01492428 \\ https://hal.science/hal-01492428}

Submitted on 19 Mar 2017

HAL is a multi-disciplinary open access archive for the deposit and dissemination of scientific research documents, whether they are published or not. The documents may come from teaching and research institutions in France or abroad, or from public or private research centers.
L'archive ouverte pluridisciplinaire HAL, est destinée au dépôt et à la diffusion de documents scientifiques de niveau recherche, publiés ou non, émanant des établissements d'enseignement et de recherche français ou étrangers, des laboratoires publics ou privés. 


\title{
Fast and Accurate Multiplicative Decomposition for Fringe Removal in Interferometric Images
}

\author{
Daniel-Chen Soncco, Clara Barbanson, Mila Nikolova, Senior Member, IEEE, Andrés Almansa, Member, IEEE, \\ Y. Ferrec
}

\begin{abstract}
Airborne Hyperspectral images can be efficiently obtained with imaging static Fourier transform spectrometers. However, to be effective on any location, this technology requires to know the relief of the scene. This is not a straightforward process, as the horizontal interference fringes on the images, which are necessary for spectrum construction, prevent efficient stereoscopic processing. We present a novel variational model for multiplicative image decomposition to separate the fringes from the panchromatic image of the scene. This multiplicative model is much more physically accurate than previous additive decomposition models inspired by cartoon-texture decomposition. It combines fully smoothed total variation operators and 1D Fourier transform. Smoothed total variation is adopted to avoid staircasing artifacts caused by traditional total variation regularisation. The use of a 1D Fourier transform is suggested by the geometry of the fringes, in order to circumvent the lack of horizontal periodicity in the interferometric pattern. We also present an optimization algorithm. Finally, a second algorithm is introduced, whose convergence is not mathematically guaranteed. However it systematically approaches the solution of the first one in much less computation time. Our experimental evaluation on real and simulated images shows that the proposed model separates fringes from the panchromatic image very accurately and that this accuracy significantly improves subpixel stereo matching results.
\end{abstract}

Index Terms-computational hyperspectral imaging, interferometric imaging, inverse problems, multiplicative image decomposition, image restoration, nonconvex variational models, bi-convex optimisation, proximal algorithms, multi-view stereo

\section{INTRODUCTION}

$\mathbf{H}$ YPERSPECTRAL imaging is a much sought-after technology, as it provides both detailed spatial and spectral information from the object of interest, with a spectral range that may go, depending on the instrument, from ultraviolet to thermal infrared. Such technology is widely used in Earth sciences; for instance, geology employs it on airborne or spaceborne vehicles for remote sensing [1], where infrared wavelengths are especially useful for prospection [2], [3], soil composition determination [4], [5] and even gas plumes analysis in vulcanology [6]. Other gases such as water vapor for atmospheric research can be identified [7]. Visible and near-infrared are used in hydrology to assess water contamination [8] and, in relation to agriculture, to identify different

D. Soncco and M. Nikolova are with CMLA, CNRS and Ecole Normale Supérieure de Cachan, Université Paris-Saclay, 94235 Cachan, France. (email chen.soncco@gmail.com, nikolova@cmla.ens-cachan.fr )

C. Barbanson and A. Almansa are with LTCI, CNRS \& Telecom ParisTech.

A. Almansa is with MAP5, CNRS UMR 8145, Université ParisDescartes, Sorbonne Paris Cité, 75720, Paris, France. (email andres.almansa@parisdescartes.fr)

C. Barbanson and Y. Ferrec are with ONERA,91123 Palaiseau, France. (email \{clara.barbanson,yann.ferrec\}@onera.fr) crop fields and monitor water use [9]. Biology is also wellknown for using infrared bands for vegetation classification [10]. Outside Earth sciences, applications also include urban planning [11] and military intelligence [12]. Finally, elevation data are sometimes coupled with hyperspectral imagery to improve detection and analysis [13], [14]

Imaging static Fourier transform spectrometers (ISFTS) are a family of imagers which deliver hyperspectral data. They offer a series of interesting advantages, including large photon collection capability, that allow for a small ground sampling distance, and sturdy optical systems. These instruments can be operated from ground [15], [16] to space [17], but they are more often airborne systems, like Lasis, developed by the Xi' an Institute of Optics and Precision Mechanics [18], that operates in the visible domain, Sieleters, developed by Onera [19], in the midwave infrared (MWIR) and the longwave infrared (LWIR) domain or THI, developed by the University of Hawai'i [20], in LWIR.

The optical design of ISFTS is based on the association of an imaging system with a lateral shearing interferometer (apart from a few instruments employing a wedge interferometer). The incident beam emitted from the observed scene therefore proceeds through the interferometer, where it is split into two paths. Two identical images are produced, but they later interfere at a varying optical path difference (OPD) thanks to the interferometer. The resulting image is that of the overflown scene on which interference fringes are superimposed, as shown in Fig. 1.

On the figure, the OPD is different on each row of the image: the fringes are oriented perpendicularly to the linear flight path. Furthermore, the established OPD values do not change over time, which is why such an interferometer is qualified as static. The fringes are thus still. For this reason, spectrum acquisition is not snapshot, but temporal: it is the motion of the aircraft which ensures that any element in the observed scene is seen through different interference states (at different vertical positions in the image). A dense sequence of images, where each one is translated from the former one by about 1 pixel, is required for this purpose. Then, matching each scene element through the sequence of images constructs an interferogram, where each value corresponds to the quantity of detected light for each optical path difference. Computing the Fourier transform of the interferogram gives access to the spectrum for the corresponding element. This process is summarised in Fig. 2.

However, in presence of non-flat scenes (containing for example buildings or natural slopes), artifacts may appear as a consequence of changing the point of view during acquisition. 
For instance, due to parallax, elevated objects appear to move at a faster speed than the elements of lesser height. Without a precise estimate of the scene relief, the position of an elevated object across the sequence of images is miscalculated and matching errors occur. The resulting interferograms may then be erroneous, as shown in Fig. 3.

As a result, an accurate estimation of ground elevation is needed in order to identify and correct flawed interferograms. A Digital Elevation Model (DEM) can be achieved by stereoscopy techniques. However, the fringes are a hindrance to stereoscopic methods. The fact that they remain at the same position on all images interferes with relief retrieval algorithms, which exploit the changes between images taken from different positions. Moreover, the fringes induce variations in radiometry that are uncorrelated to relief. As a consequence, before proceeding with relief estimation, the fringes need to be removed from the images. This problem is the topic of this article: how can one remove the interference fringes while best preserving the details of the underlying panchromatic image?

A similar problem has been studied in [21]: in this paper, based on images from Lasis, the authors also aim at separating fringes from background, but with the view of image compression. To do this, they use an additive model, with a further one-dimensional total variation (TV) regularization to suppress the residual fringes from the background image. However, the images presented in [21] to illustrate their algorithm have a very smooth texture. In this particular setting an additive image model is sufficient, but not in the more general setting we are interested in, which includes irregular textures and contrasted edges. The problem of fringe separation has also been studied in a more general context by [22], there again with an additive model, and using an adaptive norm to deal with spatial variations of the fringes orientation or frequency. Applications are various, for instance fingerprint analysis or 3D-imaging by strucured-light projection [23].

The main contribution of our paper consists in the use of a multiplicative model for image decomposition. This model is a better approximation of images obtained by an ISFTS than an additive model. It provides outstanding results for separating the fringes from the image of the scene. The paper is arranged in the following manner. Section II presents the model for interferometric image formation and related work on the texture removal problem. Our proposed solution is presented in Section III. It is declined in two different algorithms using a multiplicative image model. The advantages and drawbacks of the different solutions are also discussed. Section IV presents experimental results and gives a comparison of the various solutions in terms of stereoscopic performance. Finally, conclusion and future work are exposed in Section V.

\section{PRELIMINARIES}

\section{A. Observation model}

The primary objective of our hyperspectral interferometric system is to measure the spectral radiance $L(x, y, \sigma)$ coming from a point $(x, y)$ in image or ground coordinates in a domain $D$ and at a particular wavenumber $\sigma$. Color image sensors measure $L(x, y, \sigma)$ for three wavenumbers $\sigma$ corresponding to

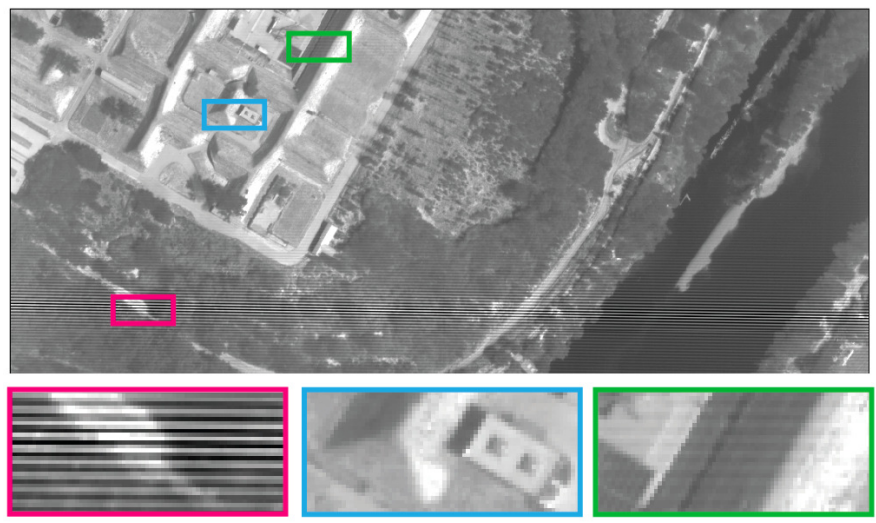

(a)

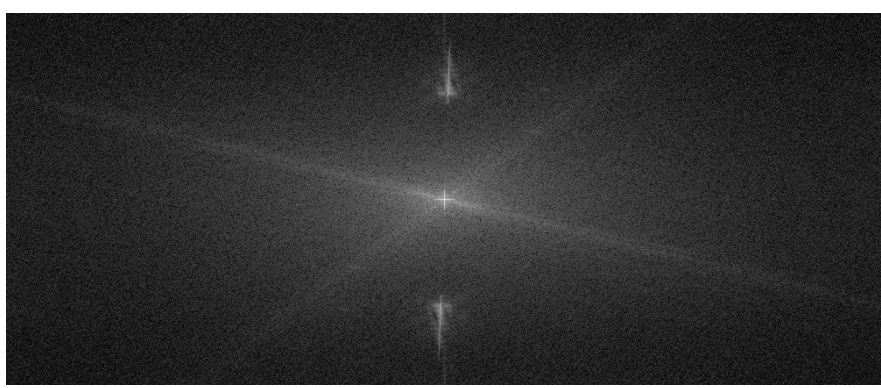

(b)

Fig. 1. On top, an image obtained with Sieleters. Subimages are magnified to show the interference fringes overlaid on the scene. In red, an area with highly contrasted fringes. In blue, an area with blurred fringes. In green, an area with slightly contrasted fringes. The area with high contrast fringes is limited to the area around the zero optical path difference, due to the broadband observed spectra. Nonetheless, fringes are actually present over the whole image. Image (b) shows the Fourier spectrum of picture (a). The frequencies of the fringes are clearly seen as two very bright vertical lines. These frequencies constitute an area we call $\Omega_{0}$
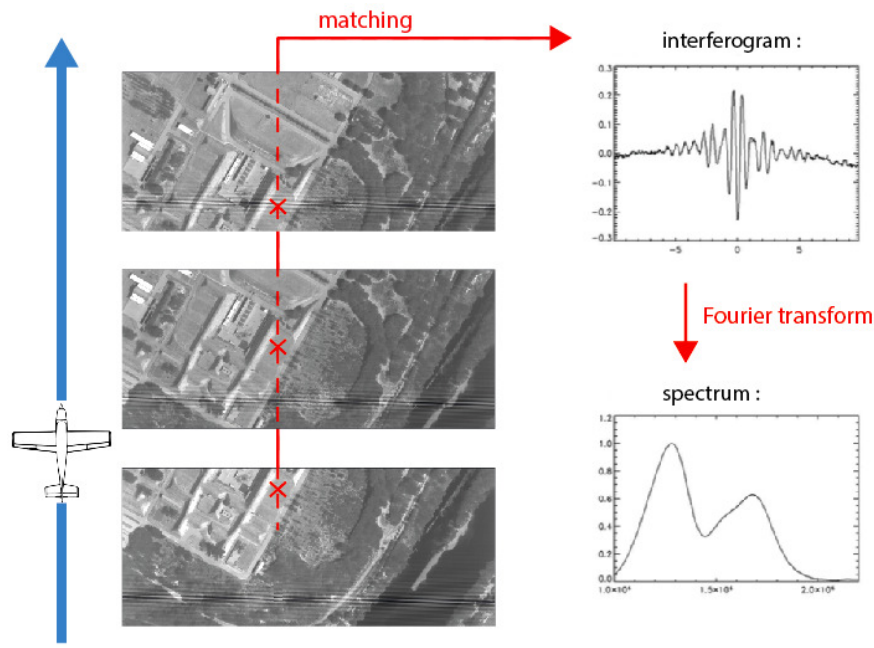

Fig. 2. Image processing flow of an airborne static Fourier transform spectrometer. All elements of the scene, like the one pointed out by the red cross on each picture, are matched through the sequence of images to build their corresponding interferogram as a function of optical path difference. Computing the Fourier transform provides the spectrum.

the red, green and blue wavelengths by means of optical filters that only let the desired wavelength intervals through. In hyperspectral imaging, where we do not only want to distinguish 

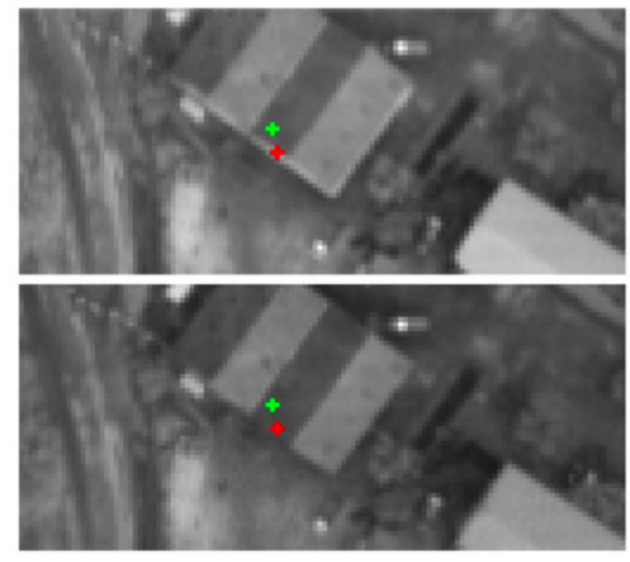

(a)

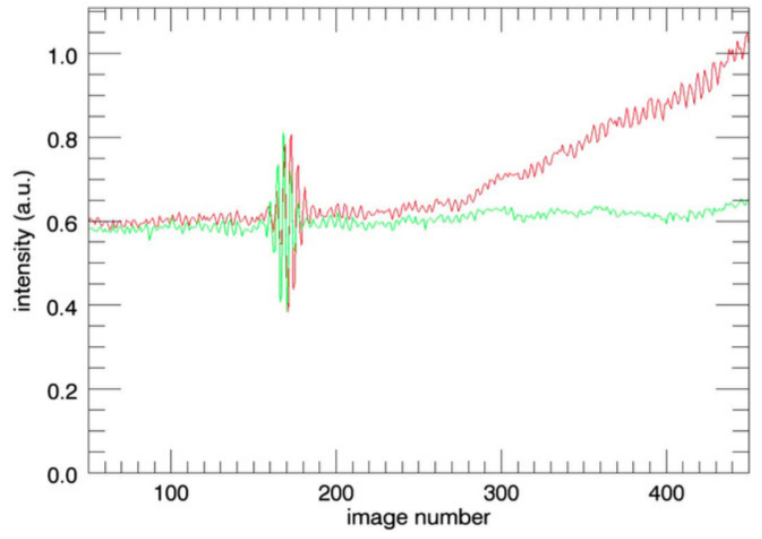

(b)

Fig. 3. On Fig. a, two images of a sequence acquired by the ISFTS CaHyD [24] are shown. Values from matching scene elements are used to construct the interferogram, as on Fig. b. The crosses on the images correspond to matches made without taking the height and geometry of the building into account. Hence, in the case of the red cross, part of the rooftop and part of the wall are used to construct a flawed interferogram. The green crosses are also mismatched, but are contained in an homogeneous area, thus providing a correct interferogram.

three, but hundreds of different wavelengths, filtering methods can be substituted by interferometers.

The geometry of the lateral shearing interferometer [25] shows that the acquired image $w(x, y)$ is related to the apparent spectral radiance ${ }^{1} L$ by:

$$
w(x, y)=\int L(x, y, \sigma)(1+F(x, y, \sigma)) d \sigma
$$

where $\sigma$ is the wavenumber and $F$ introduces the fringe oscillations in the acquired image $w(x, y)$. More precisely,

$$
F(x, y, \sigma)=C(\sigma) \cos (2 \pi \delta(x, y) \sigma+\phi(\sigma))
$$

where $C$ is a contrast term, $\delta(x, y)$ is the optical path difference (OPD) and $\phi$ accounts for phase perturbations. In ISTFS, $\phi$ has a little influence and the OPD is nearly planar,

$$
\delta(x, y)=a x+b y+\varepsilon(x, y)
$$

${ }^{1}$ By apparent we mean that the sensitivity of the detector is included in this term. where the variations of $\varepsilon$ are known to be very small. Without loss of generality, we consider that $a \gg b$. Consequently, the fringes are nearly horizontal straight lines as seen in Fig. 1. From (2), the wavenumber $\sigma$ creates fringes at the spatial frequencies $-\sigma(a, b) \cup \sigma(a, b)$ (assuming a flat scene). The sensitivity of the imaging device is given by an interval $\left[\sigma_{\min }, \sigma_{\max }\right]$. Then the fringes are contained in the spatial frequency domain $\Omega_{0}$ :

$\Omega_{0}=\Omega_{0 x} \times \Omega_{0 y}=\left\{-\sigma(a, b) \cup \sigma(a, b): \sigma \in\left[\sigma_{\min }, \sigma_{\max }\right]\right\}$

The goal is to extract the fringe pattern introduced by $F$. Based on (1), two tractable simplifications can be derived.

a) Additive model: The simplest way to approximate the model in (1)-(2) is given by

$$
w(x, y)=u(x, y)+v(x, y)
$$

where

$$
\begin{aligned}
u(x, y) & =\int L(x, y, \sigma) d \sigma \\
v(x, y) & =\int L(x, y, \sigma) F(x, y, \sigma) d \sigma
\end{aligned}
$$

where $u$ is the sought-after panchromatic image and $v$ is the fringe pattern image.

If we now assume that the scene $u$ only contains smooth objects, then, in the spatial frequency domain, the Fourier transform $\hat{v}$ of the fringe pattern will be limited to a small area $\Omega$ around $\Omega_{0}$.

b) Multiplicative model: Assuming that all points in $D$ have the same spectral response $B(\sigma)$, one has :

$$
L(x, y, \sigma)=B(\sigma) I(x, y)
$$

By setting the constant $B_{t o t}:=\int_{\sigma} B(\sigma) d \sigma$, we obtain from (1)

$$
w(x, y)=I(x, y) B_{t o t}\left(1+\frac{1}{B_{t o t}} \int B(\sigma) F(x, y, \sigma) d \sigma\right)
$$

We then get the following image model :

$$
w(x, y)=u(x, y)(1+v(x, y))
$$

where the panchromatic image $u$ and the fringe image $v$ are given by :

$$
\begin{aligned}
u(x, y) & =I(x, y) B_{t o t} \\
v(x, y) & =\frac{1}{B_{t o t}} \int B(\sigma) F(x, y, \sigma) d \sigma
\end{aligned}
$$

Since $B$ does not depend on $(x, y)$, and $F$ only has frequencies contained in $\Omega$, we can deduce that the spectral support of $v$ should be exactly limited to $\Omega_{0}$. Nevertheless, with real images, our hypothesis that all points have exactly the same spectrum is obviously imprecise, and that is why we still have to enlarge $\Omega_{0}$. The finite size of the images also leads to a broadening of $\Omega_{0}$. 
c) Additive versus Multiplicative model: Even though it is still an approximation, the multiplicative model matches much better the actual image formation than the additive one. Indeed, the hypothesis for the latter is quite strong, although it does not appear at first glance when looking at Fig. 1b. From this figure, it is clear that the low spatial frequencies are much more significant than high spatial frequencies. However, on the one hand, depending on the observed scene, or on the area of the observed zone, the content in medium or high spatial frequencies can be much higher. This is for instance the case on Fig. 1b, where we can note that some directions are favoured. For this image, these directions are far enough from the one of the interference fringes, but for other scenes, there could be an overlap with the $\Omega$ domain assigned to the interference fringes. On the other hand, the image details at a fine scale are often very useful. This is especially our case, since, as mentioned in the introduction, our further goal is to perform stereoscopy, for which the information content at the pixel scale is quite useful. Thus, the additive model may lead to inadequate results, as it will be shown in the next subsection. Regarding the multiplicative model, we first note from equation (1) that, at each wavenumber, there is indeed a multiplication between the scene radiance and the interference pattern. The assumption that all points have the same spectrum is then obviously a simplification, but it is verified at first order for remote sensing images. The first reason, which is quite basic, is that the apparent spectrum of all the points is limited by the spectral sensitivity of the instrument. Besides, the atmospheric transmission and radiance is also shared by all the observed points. Lastly, even if there are spectral variations in natural scenes, we never have very sharp differences between the spectra. Therefore, the multiplicative model is more appropriate for our framework. However, it leads to non-convex restoration models, which are numerically difficult to deal with. This is why the common approach in the literature is to use the simpler additive model, which has the flaw of requiring a much larger dilation of $\Omega_{0}$ than the multiplicative one. Furthermore, in regions where the image contains sharp and highly contrasted edges, the spectral support assumption for $\mathrm{v}$ is no longer valid and additive models introduce artifacts, as it will be seen in the next subsection.

\section{B. Related work}

Decomposing an image into an oscillatory and a structure components has been intensely explored through the cartoontexture model [26]. Such a decomposition aims at separating an image in the following manner: $w=u+v$, where $u$ is smooth and $v$ contains the oscillating patterns of the image. This is done by representing $u$ as a smooth function of bounded total variation and $v$ by an object of a Hilbert space. The resulting minimisation problem is of the form:

$$
\inf _{u} \int|\nabla u|+\lambda|| w-u \|_{\mathcal{H}}^{2}
$$

Other representations for $v$ have been proposed, depending on the properties that are sought. [27] suggests that the texture and structure are not correlated, and minimises the oscillating term using a correlation criterion. [28] determines the structure and texture by identifying them with low-pass and high-pass components respectively.

In the case of interference fringe removal, the desired result is not a smooth image, but an image without fringes, keeping the texture and the edges in the scene. Inspired from cartoontexture decomposition, the idea in [29] is to apply an additive model to the measured images $w$, where this time $u$ is the image of the scene not perturbed by fringes, and $v$ contains solely the interference fringes. It provides an approximation that allows for a simple convex optimisation scheme.

As explained in II-A, the frequencies of the fringes are mainly contained in a small area, called $\Omega$. Only the frequencies inside $\Omega$ are considered to be altered by fringes, so the rest of the spectrum is kept intact. To proceed, the total variation operator [30] is used. It allows to smooth the image by penalising oscillations and is also useful to extrapolate the frequencies of the spectrum inside $\Omega,[31],[32]$. This procedure is expressed in the following convex minimisation problem, as presented in [33]:

$$
\inf _{u} T V(u) \quad \text { subject to } \hat{u}_{\left.\right|_{\Omega^{c}}}=\hat{w}_{\left.\right|_{\Omega^{c}}}
$$

where the symbol "hat" stands for Fourier transform. Although the results are good for many images, residuals can remain in areas containing sharp contrasted edges, see, e.g., Figs. $4 \mathrm{a}$ and $4 \mathrm{~b}$. This image was acquired when a building with a periodic pattern was in the area of the highly contrasted fringes. In the Fourier domain, such a building creates a DC term, a fundamental and multiple harmonics, all aligned along a roughly horizontal direction. The interference fringes $v$ then act as a modulation term, with the carrier frequency inside the $\Omega$ domain (in white on Fig. 4d). Thus, each frequency of the building pattern is replicated (see Fig. 4c or Fig. 12b). When applying the additive model, only the $\Omega$ domain (the white rectangle on Fig. 4d) is modified. Consequently, only the replica of the DC term is regularized, but not the replicas of the fundamental or of the harmonics, which creates artifacts in the final image (Fig. 4b). Moreover, since these replicas are retained, the TV regularization creates spurious information inside $\Omega$. This is visible on Fig. 4c, or on Fig. 12c.

The fact that the scene is modulated by the frequency of the fringes corresponds, in the spectral domain, to a convolution of the interference term by the frequencies of the scene. Therefore, if the observed scene contains elements with high gradients, frequencies of the fringes will be found not only on the lower frequencies of the spectrum where $\Omega$ is set (Fig. 4d), but also on higher frequencies, as is shown in Fig. 4c.

One possibility is to use a larger domain $\Omega$, but this is at the expense of the loss of some high frequency details in $u$. A better solution is to adopt a physically more accurate multiplicative image model as explained in Section III. Multiplicative data-fitting along with TV regularization has been considered in the context of Poisson denoising (see [34]); in the quite different context of fringe removal the solution proposed here is one-of-a-kind.

Another issue concerns the discrete total variation operator used and the 2D-Fourier transform, which are bound to produce artifacts such as staircasing, [35]. All in all, this scheme 


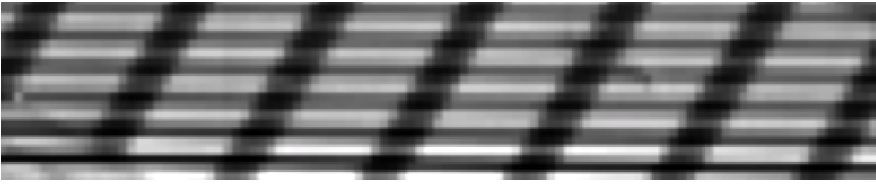

(a) Extract from the measured image

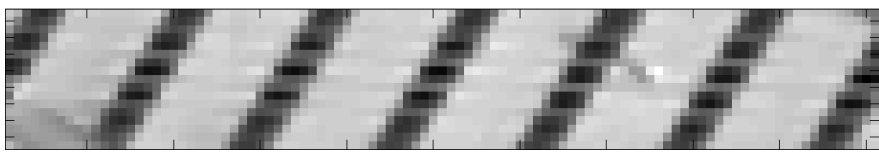

(b) Extract from the regularised image

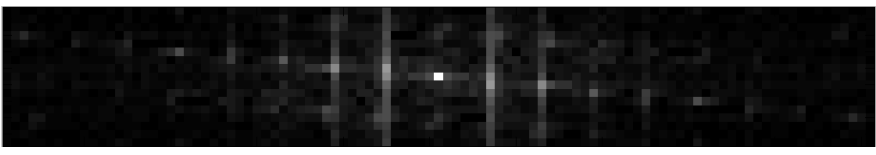

(c) Fourier transform associated to (b)

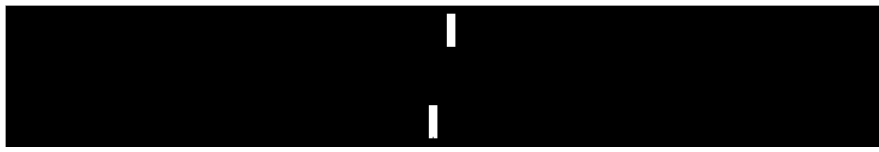

(d) $\Omega$ region associated with (a)

Fig. 4. Extract from an image with fringe residuals. The fringes correspond to the horizontal stripes. It can be seen that most have been removed in image (b). Yet, some remain on the objects with high radiometric variations. These correspond in (c) to the harmonics of the fundamental frequency which lay outside $\Omega$.

is not accurate enough but provides an example of mainstream algorithms for comparison in Section IV.

\section{Main contributions}

In this work we propose a new variational approach for removing fringes from the images acquired by an ISFTS. Our approach introduces key ingredients that have a radical implication on the restored image. A summary is given next.

- Our method is robust with respect to sharp edges. In comparison with usual additive models, our multiplicative model has important advantages: it is much more accurate when restoring regions near sharp edges - a scenario where additive models cannot clearly separate the fringes $v$ from the panchromatic image $u$.

- Fringe frequencies estimation. Since the quality of the image restoration critically depends on the accuracy of the fringe pattern frequencies, we provide a simple and robust algorithm for their estimation based only on the image content.

- Oracle estimation. The spectral constraint on $v$ is enforced only in the direction orthogonal to the fringes. Compared to two-dimensional constraints, the onedimensional constraint alleviates some possible artifacts arising from the DFT approximation. Furthermore, using the fringe pattern frequencies for an observed scene $w$, we reconstruct quite a faithful oracle image which is used as initialization in the subsequent algorithms.

- Frugal regularization. Our variational model is composed out of a multiplicative data term, a spectral penalty on the fringes along with a smoothed TV term on the rows of the observed image and on the columns of the fringe pattern which is more efficient in our setting and less expensive to compute than a standard two-dimensional TV regularization.

- Absence of staircase artifacts. The smoothed TV avoids the well-known staircase artifacts (commonly associated to non-differentiable TV minimization) that are particularly damaging for subpixel stereo correspondence of the resulting restored images.

- Convergent algorithm with explicit proximal iterations. Our model is biconvex and we alternate iterations with proximal functions which, explicitly computed, become simple.

- Simple filtering algorithm. We provide a simple and fast algorithm for which no convergence proof is available. An extensive experimentation on a large dataset shows that the result of the fast algorithm is always extremely close to the solution of the bi-convex multiplicative model.

\section{DESCRIPTION OF OUR METHOD}

Assuming the multiplicative model, the relationship between the measured image $w$ and both the panchromatic image $u$ and the fringe image $v$ can be summarized as

$$
w=u_{\circ}(1+v)+\eta
$$

where 。 stands for the Hadamard (componentwise) product and $\eta$ accounts for noise perturbations.

The method presented in this work was optimized on a set of nine ground-truth images where the decomposition into fringes component $v$ and panchromatic component $u$ is known exactly.

\section{A. Nine ground-truth panchromatic images}

To obtain noise-free simulated data, we proceeded in the following way. We used two co-registered high resolution infrared images, to deduce, for each pixel, a temperature and an emissivity. Note that these values are neither the actual temperature nor the true emissivity of the pixels, however they are realistic values, which is quite enough. From these temperature and emissivity maps, and taking into account the Planck's law and a standard atmosphere transmission and selfemission, we computed a spectral cube, corresponding to the spectral radiance at entrance pupil level. This spectral cube was then converted to a high resolution interferometric image, based on instrumental characteristics of Sieleters-MWIR (optical path difference map, spectral sensitivity, contrast and phase) and on equations 1-2. Lastly, this image was spatially filtered and downsampled, to obtain an image with the same size and resolution as Sieleters-MWIR. We thus obtained a set of images, based on the physical model. We later refer to these images as measured images, for they simulate the images obtained with an ISFTS. We also computed a panchromatic image, by suppressing the interference term in equations $1-2$, and a fringe image, from the average spectrum of all objects in the scene. The panchromatic image will later be called ground truth, as it is true enough to be used as such. One example is 

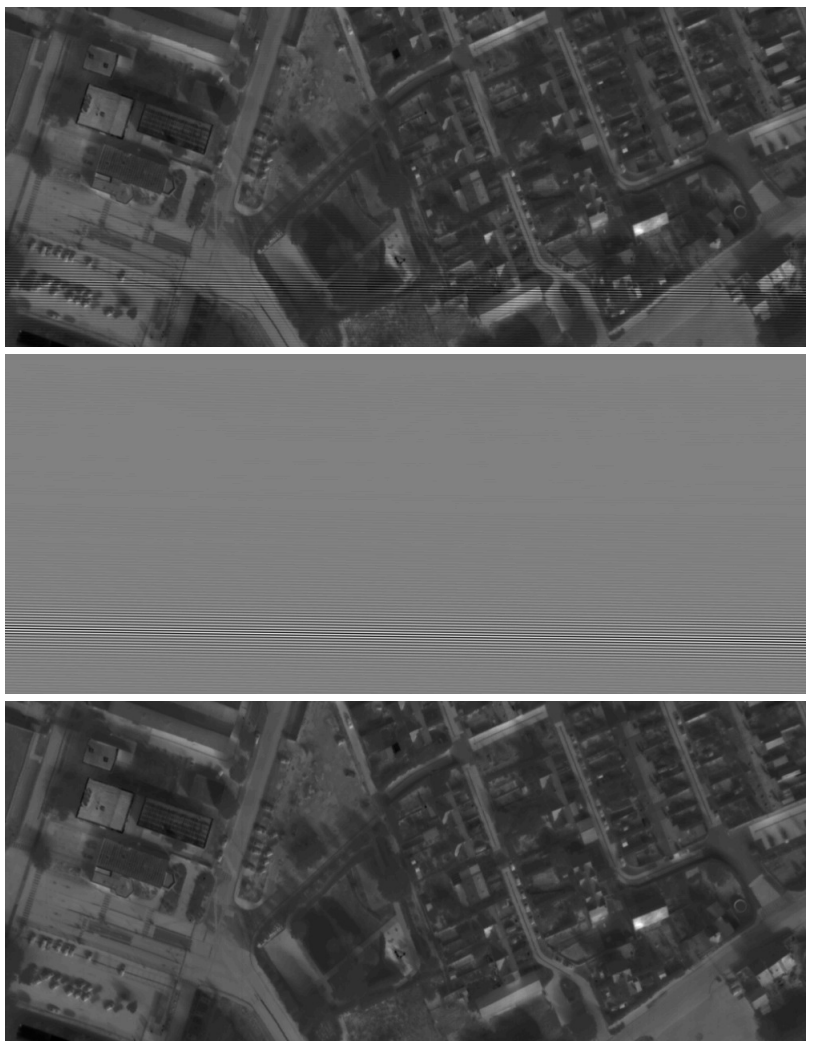

Fig. 5. Simulated data. Top: measured image. Middle: fringe pattern. Bottom: ground truth.

given in Fig. 5. These images are given on a discrete grid of size $m \times n$ where $m=424$ and $n=1000$. In these images, the noise perturbation can be neglected, i.e., $\eta \approx 0$ in (11).

\section{B. Notation}

For a $m \times n$ matrix $u$, its rows are denoted by $u_{i, *}$ and its columns by $u_{*, i}$. We use the vertical mirror function $M: \mathbb{R}^{m \times n} \rightarrow \mathbb{R}^{3 m \times n}$ given by $M u=(\bar{u}, u, \bar{u})^{T}$ where $T$ stands for transpose and $\bar{u}$ is the matrix consisting of rows $m, m-1, \ldots, 1$ of $u$. Its left-inverse, denoted by $M^{\dagger}$, extracts $u$ from $z=M u$ by taking rows $m+1, \ldots, 2 m$ of $z . I_{m}$ is the identity operator on $\mathbb{R}^{m}$. A vector or an image with ones of arbitrary dimension will be denoted by 1 . The notation $\mathcal{F}$ will signify the Hamming windowed 1D Discrete Transform Fourier applied to the columns an image.

\section{On the Fourier domain constraint}

Our focus is on methods that constrain the Fourier transform of the image to vanish on the frequencies interval produced by the fringe pattern.

The fringe pattern $v$ is nearly horizontal, typical of interferometric images. The 1D Fourier transform of the rows of the measured image $w$ are not expected to change a lot except for some low frequencies. Thus the 1D Fourier transform of the rows of the measured image $w$ and the rows of the ground truth $u$ should be nearly the same; see Fig. 6 left and middle. The main difference is in the 1D Fourier transforms of the columns of the ground truth $u$ and the measured image $w$; see Fig. 6 right. Therefore, we focus on using only the 1D Fourier transforms of the columns.

a) Estimation of the spectral support of the fringe pattern: From the observation model in (1) and (2) it is known that the fringes are concentrated in a region delimited by $f_{\min }$ and $f_{\max }$, the frequencies of the spectral support of $v$. These can be determined analytically from the optical path difference map from Eq.(3). However, they usually shift during the acquisition. Since the restoration quality critically depends on the accuracy of $f_{\min }$ and $f_{\max }$ we propose a robust method for their estimation for each image $w$. The method is motivated by Fig. 6 and the algorithm is described next.

Algorithm 1: Estimation of $\left(f_{\min }, f_{\max }\right)$

$$
\begin{aligned}
& \begin{array}{l}
\text { INPUT: } \mathrm{w} \\
\bar{w}=\left(I_{m} \otimes\left(\frac{1}{3}, \frac{1}{3}, \frac{1}{3}\right)\right) \text { column mean }(\log |\mathcal{F}(M \circ w)|)
\end{array} \\
& \{\text { where } \otimes \text { is the Kronecker product }\} \\
& \widehat{w}=\left(\begin{array}{c}
\bar{w}_{\left\lfloor\frac{1}{2} m+1\right\rfloor}, * \\
\ldots \\
\bar{w}_{m, *}
\end{array}\right) \text { \{where }\left\lfloor\frac{1}{2} a\right\rfloor \leq a \text { is the nearest } \\
& c=\text { robust fitting }(\widehat{w}) \text { \{using a cubic curve with Cauchy } \\
& \text { weighting for parameter } 1\} \\
& {\left[f_{\min }, f_{\max }\right]=\text { the largest interval such that } c>\widehat{w}} \\
& \text { OUTPUT: }\left[f_{\min }, f_{\max }\right]
\end{aligned}
$$

The constraint set $\Omega$ is given by a binary $m \times n$ mask where the only null rows correspond to the frequencies in $\left[-f_{\max },-f_{\min }\right] \cup\left[f_{\min }, f_{\max }\right]$

b) An oracle image: Using the measured data $w$ and the estimated constraint set $\Omega$, one can build a initial oracle image $u^{(0)}$. It amounts to a filtering of the columns of $w$ :

$$
u^{(0)}=M^{\dagger}\left(\mathcal{F}^{-1}(\Omega \circ \mathcal{F}(M w))\right)
$$

and $v^{(0)}=\left(w / u^{(0)}\right)-1$, with "/ denoting the pointwise division.

\section{Variational formulation}

One notices the good reconstruction done in the oracle image $u^{(0)}$ as seen in Table I. An image showing the oracle $u^{(0)}$ is given in Fig. 15. The restoration approach is based on an inspection of the oracles $u^{(0)}$ and $v^{(0)}$ for all 9 simulated images. The main facts we notice are listed next.

(a) The columns of $u^{(0)}$ have noticeable oscillations especially in the region of the fringes; the rows of $u^{(0)}$ are very close to those of the ground truth and do not need particular smoothing. (b) The 1D DFT of the fringe columns oracle are still perturbed in the frequency range $-\left[f_{\max }, f_{\min }\right] \cup\left[f_{\min }, f_{\max }\right]$ hence we need to penalize $\Omega_{\circ} \mathcal{F}(M w)$.

(c) The rows of $v^{(0)}$ are quite more perturbed than its columns; this together with (b) leads us to apply a smoothing on the rows of $v$. 

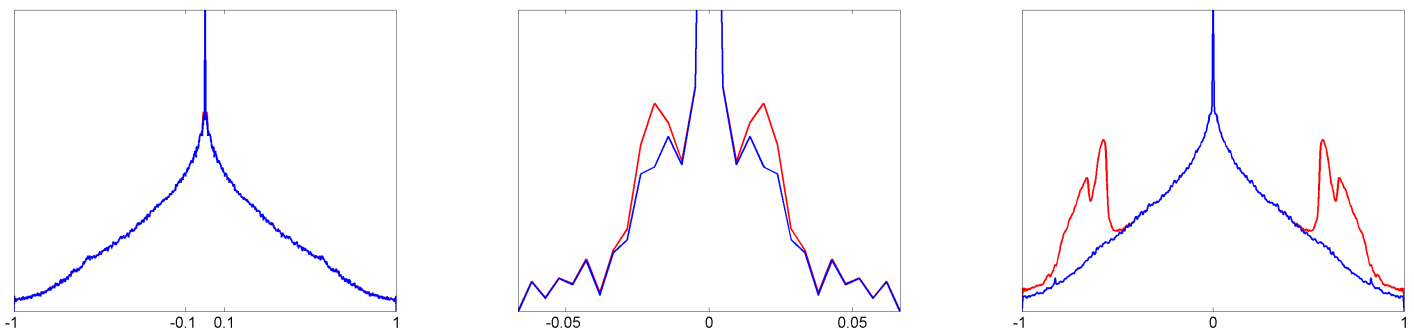

Fig. 6. Log of 1D Fourier transforms of the ground truth (blue) and the measured image $w$ (in red). The plot on the left shows the mean spectrum of rows The one in the middle is a zoom of the first around the zero frequency. The plot on the right shows the mean spectrum of columns.

(d) Finally, we want to keep close to the measured image $w$ since the noise in the multiplicative model, see (11), is very small.

Combining all these observations leads to a variational model of the form $J(u, v)$ given by

$$
\lambda \Phi_{\alpha_{1}}(u)+\Psi_{\alpha_{2}}(v)+\beta \frac{\|T(v)\|_{2}^{2}}{2}+\gamma \frac{\left\|w-u_{\circ}(1+v)\right\|_{2}^{2}}{2}
$$

where we set $T(v):=\Omega \circ \mathcal{F}(M \circ v)$ and

$$
\begin{aligned}
\Phi_{\alpha_{1}}(u) & :=\sum \varphi_{\alpha_{1}}\left(u_{i, j}-u_{i-1, j}\right), \\
\Psi_{\alpha_{2}}(v) & :=\sum \varphi_{\alpha_{2}}\left(v_{i, j}-v_{i, j-1}\right) .
\end{aligned}
$$

The choice of $\varphi$ is important. We focus on a convex edge preserving $\varphi$ [36]. A popular choice is $\varphi(t)=|t|$ which amounts to median prior [37] and to a particular discretization of the TV model. It is well known that such a $\varphi$ introduces constant segments in the column and the row estimates of $u$ and $v$, respectively, which is not desired. Therefore, we have to choose a smooth approximation of $|t|$. A frequent choice is $\varphi_{\alpha}(t)=\sqrt{t^{2}+\alpha}$, but its derivative is involved for numerical implementation. Another choice that leads basically to the same solution is

$$
\varphi_{\alpha}(t)=|t|-\alpha \log \left(1+\frac{|t|}{\alpha}\right) .
$$

Its derivative $\varphi_{\alpha}^{\prime}(t)=\frac{t}{\alpha+|t|}$ is simple to compute which saves up to $20 \%$ running time compared to the square-root function [38].

The parameters in (13) were fixed using the simulated images. It is quite obvious that $\beta$ and $\gamma$ should be large numbers by (b) and (d) because they represent "constraints". The others (quite simple from the simulated images) are given in a subsequent section.

\section{E. Minimization Algorithm}

The objective $J$ in (13) is biconvex. A common practice for solving biconvex problems is the Alternate Convex Search where $u$ and $v$ are updated alternatively by fixing one of them [39]. More precisely, our algorithm follows a proximal regularization of the Gauss-Seidel scheme, see also [40]. The objective $J$ in (13) is of the form $F(u)+G(v)+H(u, v)$ :

$$
J(u, v)=F(u)+G(v)+H(u, v),
$$

where

$$
\begin{aligned}
H(u, v) & =\frac{\gamma}{2}\left\|u_{\circ}(v+1)-w\right\|_{2}^{2}, \\
F(u) & =\lambda \Phi_{\alpha_{1}}(u), \\
G(v) & =\beta \frac{\|T(v)\|_{2}^{2}}{2}+\Psi_{\alpha_{2}}(v) .
\end{aligned}
$$

Let $D_{h}$ and $D_{v}$ denote the horizontal and vertical difference operators on $m \times n$ images. Using vector notation, one has

$$
\begin{aligned}
& \nabla F(u)=\lambda D_{v}^{T} \varphi_{\alpha_{1}}^{\prime}\left(D_{v} u\right), \\
& \nabla G(u)=\beta T^{T} T v+D_{h}^{T} \varphi_{\alpha_{2}}^{\prime}\left(D_{h} v\right),
\end{aligned}
$$

which gradients are used in the forward step in Algorithm 2 given next.

This algorithm is initialized with the oracle image $u^{(0)}$ and the corresponding $v^{(0)}$. For practical reasons the algorithm below is applied with $w$ normalized as in steps (a)-(b) below. This means we apply Algorithm 1 with the normalized input $w$ to obtain the corresponding values $f_{\min }$ and $f_{\max }$. Further, $\tau_{1} \lesssim \frac{2}{\operatorname{Lip} \nabla F}$ and $\tau_{2} \lesssim \frac{2}{\operatorname{Lip} \nabla G}$, see [40]. The Lipschitz constants here are calculated as the upper bounds of $\nabla^{2} F$ and of $\nabla^{2} G$, respectively. Using that $\left\|D_{h}^{T} D_{h}\right\|=\left\|D_{v}^{T} D_{v}\right\| \lesssim 4$, they read as $\operatorname{Lip} \nabla F=\frac{4 \lambda}{\alpha_{1}}$ and $\operatorname{Lip} \nabla G=\beta+\frac{4}{\alpha_{2}}$.

\section{Algorithm 2}

INPUT: $f_{\min }, f_{\max }, u^{(0)}, v^{(0)}, \tau_{1}, \tau_{2}, n_{\text {iter }}, \alpha_{1}, \alpha_{2}, \lambda, \beta$, $\gamma$

(a) Compute $c_{1}:=\operatorname{mean}(w)$ and $c_{2}:=8$ s.t.d. $(w)$

(b) Normalize $w \leftarrow 1+\frac{w-c_{1}}{c_{2}} \quad u^{(0)} \leftarrow 1+\frac{u^{(0)}-c_{1}}{c_{2}}$

for $k=0$ to $n_{\text {iter }}-1$ do

$u^{(k+1)}=\operatorname{prox}_{\tau_{1} H\left(\cdot, v^{k}\right)}\left(u^{(k)}-\tau_{1} \nabla F\left(u^{(k)}\right)\right)$ \{minimize in $u$ \}

$v^{(k+1)}=\operatorname{prox}_{\tau_{2} H\left(u^{k+1}, \cdot\right)}\left(v^{(k)}-\tau_{2} \nabla G\left(v^{(k)}\right)\right)\{$ minimize in $v\}$

\section{end for}

OUTPUT: recovered image $\widehat{u}=c_{1}+\left(u^{n_{\text {iter }}}-1\right) c_{2}$

The proximity operators have a simple closed-form expression. Noticing that $\nabla_{u} H(u, v)=\gamma(v+1) \circ((v+1) \circ u-w)$ and that $\nabla_{v} H(u, v)=\gamma u_{\circ}\left(u_{\circ}(1+v)-w\right)$, one obtains

$$
\begin{aligned}
\operatorname{prox}_{\tau_{1} H(., v)}(z) & =\frac{z+\tau_{1} \gamma \circ(v+1) \circ w}{1+\tau_{1} \gamma(v+1) \circ(v+1)}, \\
\operatorname{prox}_{\tau_{2} H(u, .)}(z) & =\frac{z+\tau_{2} \gamma u \circ(u-w)}{1-\tau_{2} \gamma u \circ u},
\end{aligned}
$$

where division is componentwise. 
The convergence of the algorithm to a critical point of $J$ can be found in [41].

Remark 1. Using the normalization in (a)-(b), the new measured image (or sequence of images) has mean 1 and standard deviation 0.125. This is helpful for two reasons: (1) one can process some erroneously measured images $w$ involving negative pixels; and (2) one can fix the "free" parameters $\alpha_{1}$, $\alpha_{2}, \lambda, \beta$ and $\gamma$ so that they can be applied to any image recorded with a similar device. At the end of the iterations, this normalization is restituted in the recovered image $\widehat{u}$.

\section{F. Iterative filtering to solve the multiplicative model}

Let us have another look at the observations (a)-(d) given in section III-D. We want to use them in a different way to improve the oracle image $u^{(0)}$ in (12). In the light of (a) and (c), we want to minimize both $\Phi_{\alpha_{1}}$ and $\Psi_{\alpha_{2}}$ for the same values of $\left(\alpha_{1}, \alpha_{2}\right)$ (assuming thus that our prior modeling on $u$ and $v$ in Algorithm 2 was correct enough). Furthermore, by (d) we want to closely satisfy the data-fidelity equation in (11) and by (b) - the knowledge that the 1D columnwise Fourier transform of the fringe pattern $v$ belongs to the interval $-\left[f_{\max }, f_{\min }\right] \cup\left[f_{\min }, f_{\max }\right]$. Formally, this reads as

$$
\begin{aligned}
\operatorname{minimize} & \Phi_{\alpha_{1}}(u)+\Psi_{\alpha_{2}}(v), \\
\text { subject to } & w \approx u \circ(v+\mathbf{1}) \\
\text { and } & v=M^{\dagger}\left(\mathcal{F}^{-1}((\mathbf{1}-\Omega) \circ \mathcal{F}(M \circ v))\right) .
\end{aligned}
$$

The first constraint is inexact (approximated) and nonconvex. For the second constraint, we use the values $f_{\min }$ and $f_{\max }$ estimated by Algorithm 1 with normalized input $w$ as in (a)(b) below. We adopt an inexact gradient projection descent approach with step-sizes satisfying $\delta_{1} \lesssim \frac{2}{\operatorname{Lip} \nabla \Phi_{\alpha_{1}}}$ and $\delta_{1} \lesssim$ $\frac{2}{\operatorname{Lip} \nabla \Psi_{\alpha_{2}}}$. This is summarized in Algorithm 3.

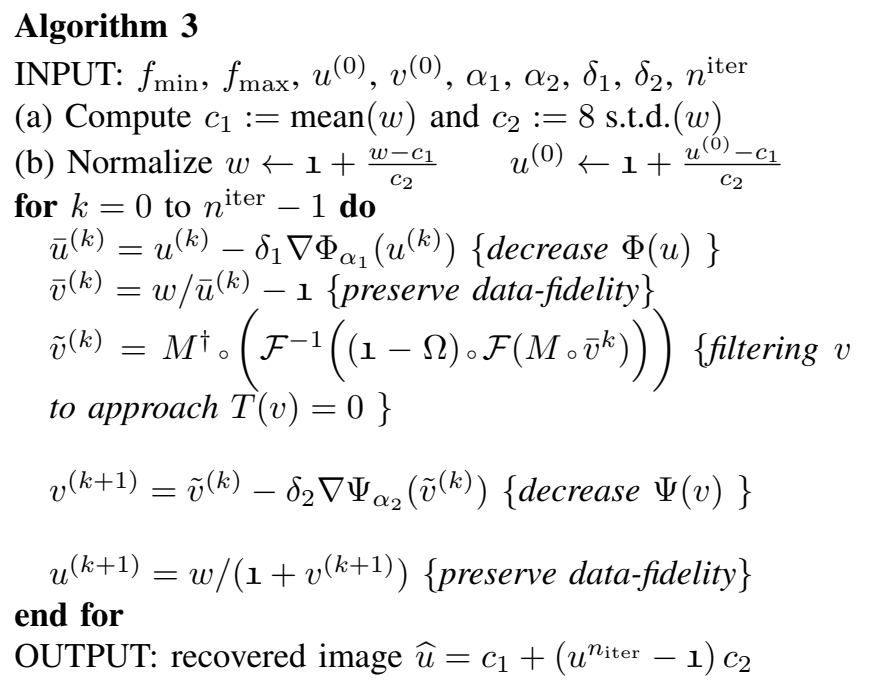

This algorithm amounts to a nonlinear filtering. We do not have guarantees for its convergence and we use it just as a filtering, a limited number of steps. This question is discussed in detail in the next subsection.

\section{G. Comparison of the Algorithms 2 and 3}

The performance of Algorithm 2 is illustrated on Fig. 7 for the image 9 (see Table 1). On the left we observe that the PSNR value at iteration 350 equals 59.6107 and at the last iteration 500 its value is 59.6524. Further increase of the iteration number would not yield much improvement. The value of the objective $J$ in (13) decreases but the decrease becomes slower. The relative error between two consecutive iterates, $\left\|u^{(k)}-u^{(k-1)}\right\| /\left\|u^{(k-1)}\right\|$ is $4.71 \times 10^{-7}$ for $k=400$ while it is $3.76 \times 10^{-7}$ for $k=500$. One can say that for $n^{\text {iter }}=500$ Algorithm 2 has nearly reached a minimum.
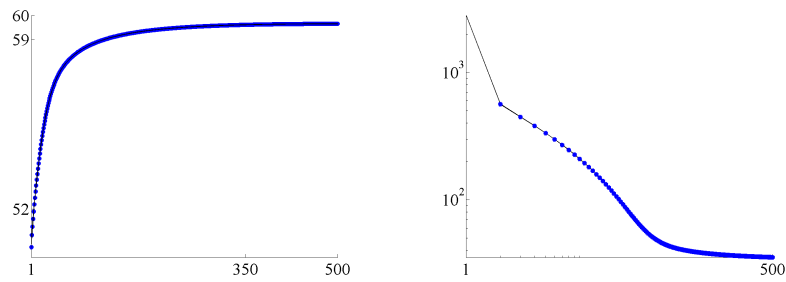

Fig. 7. Algorithm 2. Left: Increase of PSNR values with the iteration number. Right: the value of the objective $J$ in (13) in a $\log -\log$ scale.

We tested the filtering Algorithm 3 on all 9 images with ground truth, see subsection III-A, for 100 iterations. The best PSNR value occurred for iterations between 33 and 57, with a median value 41 and mean 41.44 . What is more, its decrease after the peak is slow - for 7 cases it was less then $0.5 \mathrm{~dB}$. The evolution of the PSNR for the image 9 (considered in Fig. 7) is depicted on Fig. 8 left and the relative error between two consecutive iterates on the right using a log-log scale. The PSNR value is maximum for $k=41$ and the relative error is $9.87 \times 10^{-6}$.
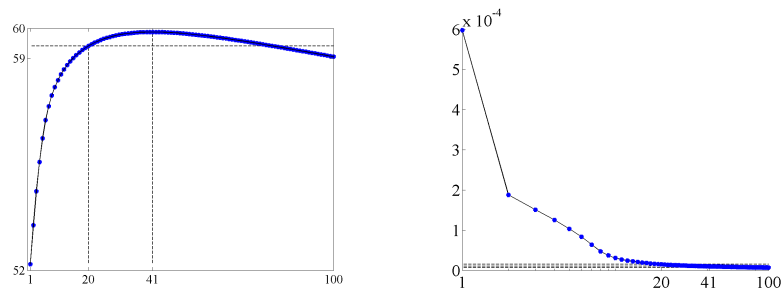

Fig. 8. Algorithm 3. Left: Evolution of the PSNR values with the iteration number. Right: The relative error between two consecutive iterates in a log-log scale.

Based on these observations, choosing $n^{\text {iter }}=20$ seems quite a safe decision. It is corroborated also by various numerical tests in section IV, as well as in the subsequent paragraph.

\section{H. Normalisation and tools for comparing the algorithms}

In many experiments we observed that starting from the Oracle initialization in (12), Algorithm 2 and Algorithm 3 yield nearly the same solution (the relative error is always less than $0.3 \%$ between the output of both algorithms as we shall see below). This observation raises important theoretical questions (when can we simplify a hard optimisation problem 
by a simple filtering-thresholding algorithm ?). This theoretical question is out of the scope of this report.

In section IV, we give some numerical evidence that the solution obtained by the algorithm 3 and by Algorithm 2 (see the objective in (13)) are nearly the same.

For this purpose, the normalization in (a)-(b) in Algorithms 2 and 3 is important. It guarantees that the images are of mean 1 and standard deviation 0.125 .

To compare the different algorithms, the relative error computed in percents is computed. We can express it as follows, with $u^{*}$ denoting the minimizer of the objective in (13) and $\hat{u}$ the result of the Algorithm 2:

$$
\varepsilon=\frac{\left\|u^{*}-\hat{u}\right\|_{2}}{\left\|u^{*}\right\|_{2}} \times 100 .
$$

The Peak Signal-to-Noise Ratio (PSNR, in $\mathrm{dB}$ ) is also computed to quantify the difference between the algorithms. It is defined as usual:

$$
\mathrm{PSNR}=10 \log _{10} \frac{\max _{x}\left|u^{*}(x)\right|^{2}}{\left\|u^{*}-\hat{u}\right\|_{2}} .
$$

For the other datasets where no ground truth is available, we directly compared the outputs of Algorithms 2 and 3.

\section{EXPERIMENTAL RESULTS}

A quantitative evaluation of the different algorithms are presented in this section. First, a comparison is made between the results in terms of image quality and running time. Then, some qualitative validations are presented and an assessment of the quality of stereoscopic processing results allows to further appraise the different methods. Using stereoscopy is motivated by the need for relief estimation for the correction of Fourier Transform spectrometers measures, which is the reason behind the process of removing fringes, as explained in Section I. Another reason that was be pointed out is that elevation models can be complementary to hyperspectral data for detection and analysis.

\section{A. Available Data}

Two types of data are used to compare results. First, the simulated data, as presented in subsection III-A.

The second type of data are experimental images acquired with Sieleters ${ }^{2}$ [19] which is a Fourier transform spectrometer. Sieleters has two channels of acquisition for infrared MWIR and LWIR. The sequences of images were obtained from an airborne platform. The plane flew at $2000 \mathrm{~m}$, resulting in a ground sampling distance of $50 \mathrm{~cm}$. The scenes describe rural and small urban areas in France in relatively flat areas.

\section{B. Parameter Settings}

The Algorithms 1, 2 and 3 presented in the previous sections require several parameters to be set. Different principles are used to choose the right parameter values for different parameter groups, namely:

\footnotetext{
${ }^{2}$ Developed by ONERA/DGA
}

- Initialization Parameters like $f_{\min }$ and $f_{\max }$ are chosen by robust fitting to training data using Algorithm 1. Once these are known the initial (oracle) values $u^{(0)}$ and $v^{(0)}$ are chosen using Equation (12).

- Numerical Parameters like $\tau_{1}=\frac{1.9}{\operatorname{Lip} \nabla F}, \tau_{2}=\frac{1.9}{\operatorname{Lip} \nabla G}$ were chosen in Algorithm 2 to be as large as possible while still ensuring theoretical convergence properties. For Algorithm 3 we do not have convergence guarantees, but by analogy to the similar Algorithm 2 we chose these parameter values $\delta_{1}=\frac{1.99}{\operatorname{Lip} \nabla \Psi_{\alpha_{1}}}, \delta_{2}=\frac{1.99}{\operatorname{Lip} \nabla \Psi_{\alpha_{2}}}$ in the same manner.

- Model Parameters like $\alpha_{1}$ and $\alpha_{2}, \lambda, \beta, \gamma$ and $n^{\text {iter }}$ should be chosen in such a way that the local minima of the variational model in equation (13) fit the ground truth solutions when available. We already know that:

- $\beta$ and $\gamma$ should have large values in order to enforce the constraints $T(v)=0$ and $w=u_{\circ}(v+\mathbf{1})$ as strongly as possible without making the optimization problem too stiff (we chose $\beta=2500$ and $\gamma=10^{4}$ );

- $\alpha_{1}$ and $\alpha_{2}$ should have quite small for better edge preservation in $u$ and $v$ (we chose $\alpha_{1}=5 \times 10^{-5}$, $\left.\alpha_{2}=5 \times 10^{-3}\right)$;

- $\lambda$ should be relatively small in order to avoid removing textures from $u$, but not too small otherwise some fringes may go into $u$ (we chose $\lambda=0.001$ );

- $n^{\text {iter }}$ should be as large as possible for Algorithm 2 to reach convergence. In practice we have found that after about $n^{\text {iter }}=500$ iterations very little was improved in terms of PSNR. For the filtering Algorithm 3 the number of iterations can have a stronger impact. We have found that optimal results where obtained for $n^{\text {iter }}=20$.

After the range of possible values for the different parameters was established according to the discussion above, the near-optimality of the chosen parameter values was checked with respect to the only Sieleters dataset, where a ground truth was available (namely the 9 images described in III-A). As it can be seen in Figures 9 and 10 neither Algorithm 2 nor 3 is sensitive to the exact value of these parameters and the PSNR is about $60 \mathrm{~dB}$. Indeed the PSNR between the solution of Algorithm 2 varied by less than $1 \mathrm{~dB}$, and $2 \mathrm{~dB}$ for Algorithm 3 when any of the model parameters was modified by a factor of 2 .

\section{Results}

Experimental results and discussion on the Algorithms 2 and 3 are presented here. A comparison between results of the additive and multiplicative image models is provided.

a) Validating Algorithm 3: Our first validation is to compare the results of Algorithm 3 (Fast algorithm) with Algorithm 2 (Optimization algorithm), because of the ensured convergence of this one. Table I shows the difference in performance and accuracy among the different algorithms applied to the 9 simulated images. Accuracy is measured in $\mathrm{PSNR}^{4}$ with respect to ground truth, whereas computational performance is measured in terms of $\mathrm{CPU}^{5}$. We observe that for all 9 simulated images the PSNR of Algorithms 2 and 3 


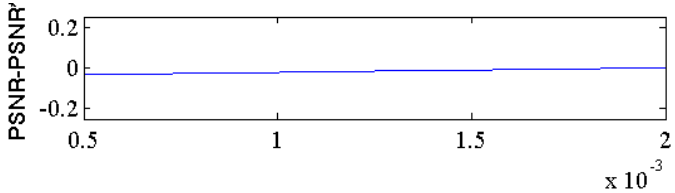

(a) $\lambda$

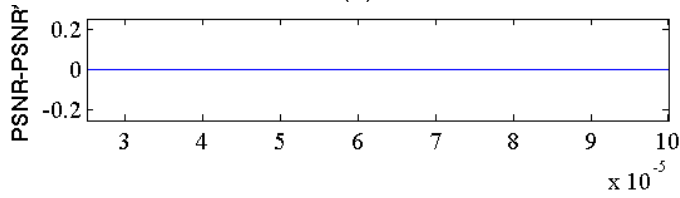

(c) $\alpha_{1}$

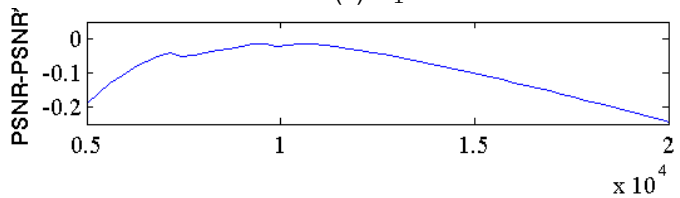

(e) $\gamma$

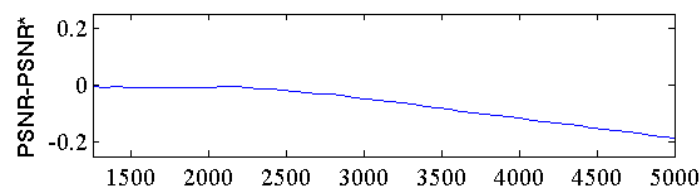

(b) $\beta$

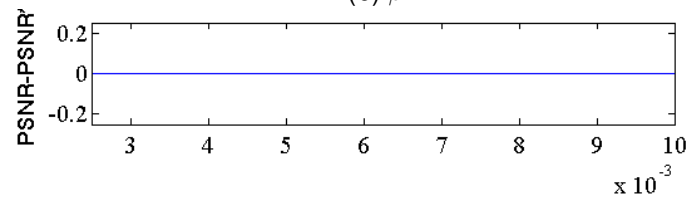

(d) $\alpha_{2}$

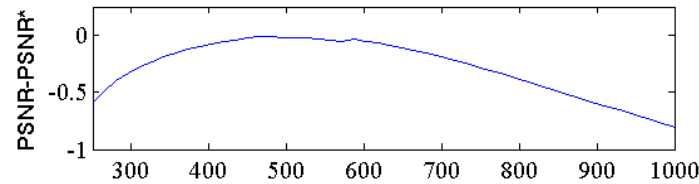

(f) $n^{\text {iter }}$

Fig. 9. Sensitivity analysis of parameters for Algorithm 2: median of psnr on 9 simulated images. The plots show the variations of PSNR when the parameters are moved around their optimal value on a range from half their value to twice their value. PSNR* corresponds to the PSNR of the optimum image found by the algorithms. Globally, variations do not exceed $1 \mathrm{~dB}$, thus showing that the final result is robust to variations in parameter values. Parameters $\gamma$, $\beta$ and the number of iterations show an optimum. This is not the case for $\lambda, \alpha_{1}$ and $\alpha_{2}$, for which barely any difference is registered.

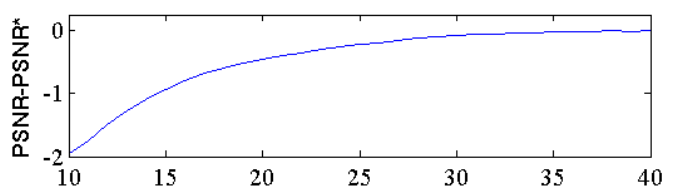

(a) $n^{\text {iter }}$

Fig. 10. Sensitivity analysis of parameters for Algorithm 3: median of psnr on 9 simulated images. The plots show the variations of PSNR when the parameters are moved around their optimal value on a range from half their value to twice their value. PSNR* corresponds to the PSNR of the optimum image found by the algorithms. The variations go up to $2 \mathrm{~dB}$, but vary very slowly after $n^{\text {iter }}=20$, our chosen value.

are nearly the same up to $0.2 \mathrm{~dB}$, meaning that their solutions cannot be more than $0.4 \mathrm{~dB}$ apart in terms of PSNR. These values thus indicated the excellent behaviour of Algorithm 3: the difference between the PSNR obtained with Algorithm 3 and with Algorithm 2 is negligible, but Algorithm 3 is more than 20 times faster.

Then, the histograms comparing the relative error $\varepsilon$ and the $\operatorname{PSNR}\left(\hat{u}, u^{*}\right)$ (following the notation in subsection III.F) between the minimizer of Algorithm 2 and the result of Algorithm 3 are presented in Fig. 11. They are computed with Sieleters images, and Algorithm 2 is used as "ground truth". The relative error appears negligible as it takes its values between $0.02 \%$ and $0.03 \%$. The PSNR takes its lower values at 70, confirming the adecuacy of Algorithm 3 in terms of image quality compared to Algorithm 2. Furthermore, on this test, Algorithm 2 was 23.2 times slower than Algorithm 3.

Finally, we propose a qualitative evaluation. Fig. 14 offers to look at results on different landscapes and textures. Fringes were successfully removed without harming image resolution and contrast. Some slight residues can however be found in the texture in Fig. 14d. This can be explained by the fact that the texture has some patterns parallel to the fringes. In
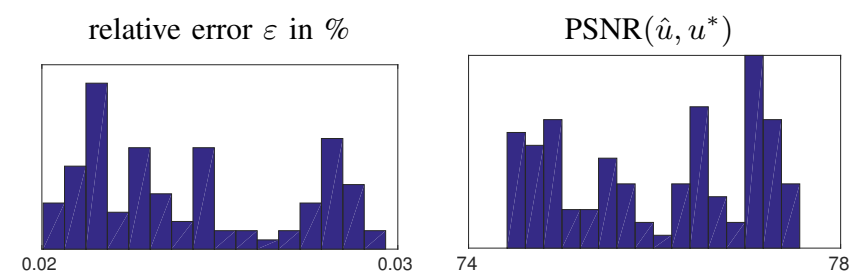

relative error $\varepsilon$ in $\%$
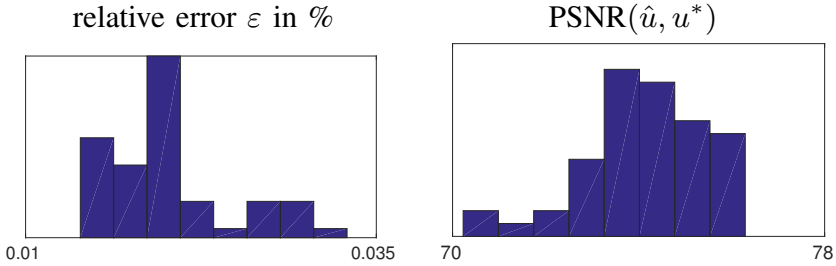

Fig. 11. Comparison between the results of Algorithm 3 with respect to the desired minimizer $u^{*}$ obtained with Algorithm 2. The first column shows histograms of the relative error as described in III.F. The second column computes the PSNR. Top line : Results are based on 100 evenly spaced images from Sieleters-MWIR. The images overlap, but it is a necessary compromise to have a large number of samples. Top line : Results are obtained with 53 disjoint (non-overlapping) images using the same instrument.

Fig. 15 images with intersecting regions were compared. The intersecting region was covered with fringes on one image, and in an area with blurred fringes on the other. Note that the latter still shows some slight fringes. We can observe that no artifact appeared and that image quality was not decreased.

b) Validating the multiplicative model: As was mentioned in subsection II-B, using an additive image formation model gives good results on many images but leaves fringe residuals on very contrasted areas (Fig. 13b). This is due to the fact that only frequencies within the small area $\Omega$ are regularised for fringe removal, when, actually, the image follows a rather multiplicative model where frequencies for the fringes are found on the whole spectrum.

If we compare the results of the additive model with the 
results of the multiplicative model of Fig. 4, extracted from Fig. 12a, it is clear that the multiplicative model succeeds in removing the impact of fringes on the periodic pattern of the building, as well the DC term as the fundamental and harmonics. Meantime, the additive model fails to remove those frequencies (Fig. 12). This explains why the multiplicative model leads to a significantly better image than the additive model (see Fig. 13).

Regarding quantitave results, Table I shows that the PSNR is indeed significantly better for the multiplicative model. What is more, the oracle gives a better scores also. This is explained by the fact that the additive model uses a 2D Fourier transform which induces artifacts, as the first and last columns do not match well due to the fact that the fringes are not perfectly aligned with the lines of the detector.

\section{Impact on Stereoscopic Performance}

As explained in the introduction, stereoscopic performance is studied as it is, in the case of Fourier transform imaging spectrometers, a likely second step for interferogram reconstruction. For this assessment we used the block matching algorithm described in [42] and the corresponding code which is available on [43].

This algorithm provides a statistical test to decide which blocks in the first image could find a statistically significant optimal correspondance in the second image. When no correspondance could be found the algorithm outputs a cyan pixel (meaning undecidable) instead of a gray-level pixel that represents the estimated disparity. As it was shown in [42], for good stereo pairs less than $30 \%$ of the pixels are undecidable, and a larger proportion of undecidable pixels means that at least one of the two images is severely corrupted with some kind of noise or does not correspond to the same scene.

As is shown in Fig. 16, the best result is clearly obtained with the images where fringes are removed using the multiplicative model associated with the 1D Fourier transform. With the measured images, where no attempt is made to erase fringes, no information of relief could be retrieved on the lower part of the image, where fringes are most contrasted. The mainstream additive model presented in II-B gives poor results, especially in homogeneous areas. As mentioned before, this can be explained by the fact that the artifacts generated from using a discrete total variation prevent the subpixel stereo algorithm from performing well.

Numerical values corroborate the visual observations, as $85 \%$ of the image could be matched with the multiplicative model, whereas this score falls to $58 \%$ and $46 \%$ for the measured image and the additive model respectively, as seen in Table II.

From these results, we conclude that the multiplicative model gives the best results, with more coverage. It therefore brings more coherence to the image and improves results on relief estimation.

${ }^{4} \mathrm{PSNR}$ values are given in $\mathrm{dB}$

${ }^{5} \mathrm{CPU}$ running times are given in seconds on an Intel QuadCore processor at $1.8 \mathrm{GHz}$ running Matlab R2016b.

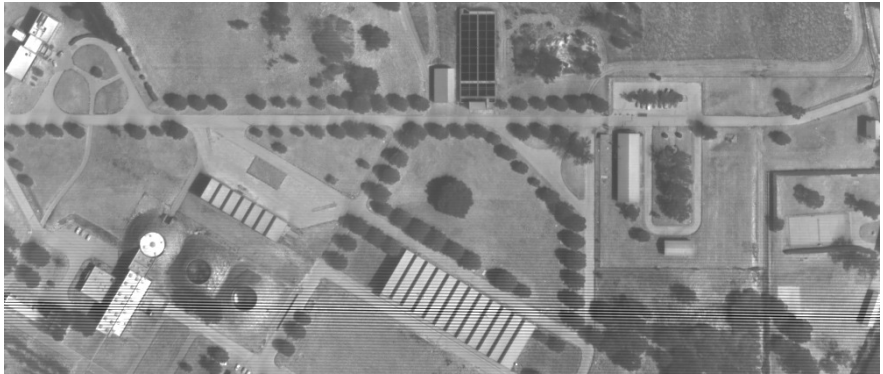

(a) Measured image from Sieleters

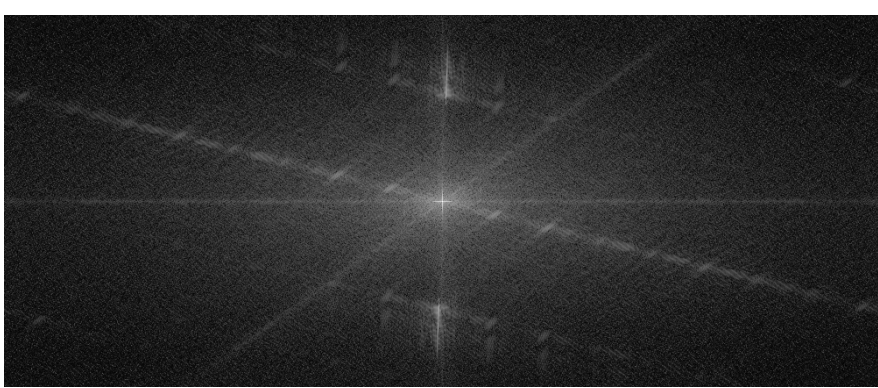

(b) Fourier transform from the measured image

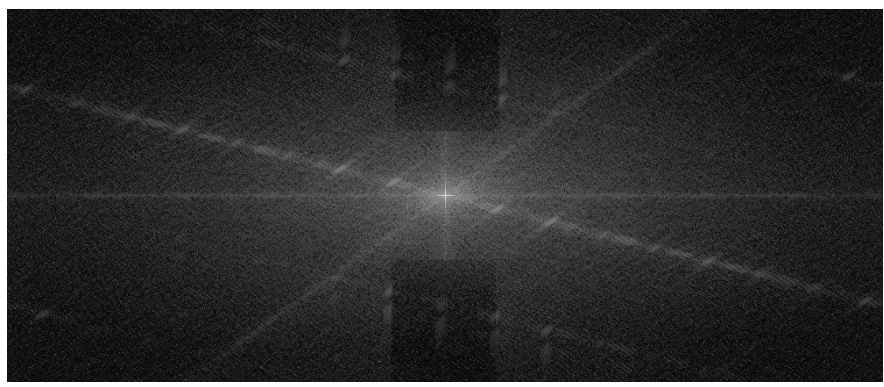

(c) Fourier transform of image obtained by additive scheme

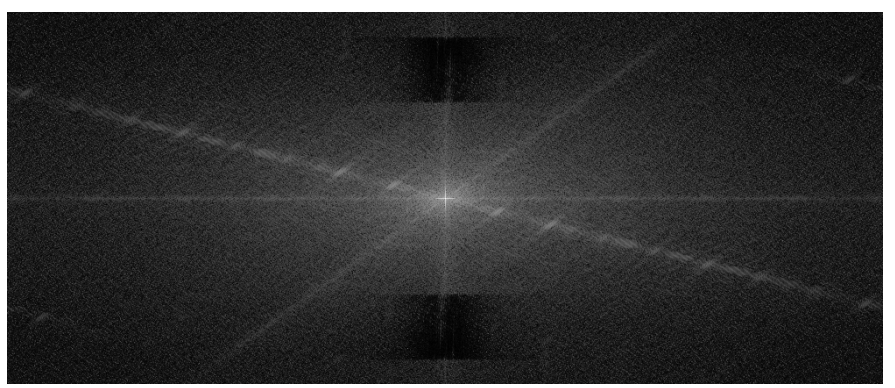

(d) Fourier transform of image obtained by multiplicative scheme

Fig. 12. Comparison between the spectra of the different schemes. On Fig. (b), the frequencies of the fringes can be seen as a very bright vertical line. Due to the image formation model, replicates of this line can be seen. The additive model, on Fig (b) manages to remove the main frequencies, but the replicas, which are outside the designated area $\Omega$, remain. The multiplicative model removes them completely, as seen on Fig. (c).

\section{CONCLUSiON}

In this paper, we have introduced a novel algorithm to remove fringes from images obtained with an imaging static Fourier transform spectrometer. This algorithm exploits a numerical model close to physical model as well as the spectral properties of interference fringes to devise operators that minimise the emergence of image artifacts during processing.

We show that it is possible to find accurate solutions to the 
TABLE I

RESULTS FROM SIMULATED DATASET WHERE THE GROUND TRUTH IS KNOWN.

\begin{tabular}{|c|c|c|c|c|c|c|c|c|}
\hline \multicolumn{2}{|c|}{ Measured image } & \multirow{2}{*}{$\begin{array}{c}\text { Oracle } \\
\text { Equation (12) } \\
\text { PSNR }^{4} \\
\end{array}$} & \multicolumn{2}{|c|}{$\begin{array}{l}\text { Fast Method } \\
\text { (Algorithm 3) }\end{array}$} & \multicolumn{2}{|c|}{$\begin{array}{l}\text { Variational Method } \\
\text { (Algorithm 2) }\end{array}$} & \multicolumn{2}{|c|}{$\begin{array}{l}\text { Additive Model } \\
\text { Equation (10) }\end{array}$} \\
\hline & $\mathrm{PSNR}^{4}$ & & $\mathrm{PSNR}^{4}$ & $\mathrm{CPU}^{5}$ & $\mathrm{PSNR}^{4}$ & $\mathrm{CPU}^{5}$ & $\mathrm{PSNR}^{4}$ & $\mathrm{CPU}^{5}$ \\
\hline Image 1 & 21.27 & 53.20 & 59.09 & 1.61 & 59.10 & 40.81 & 38.07 & 1828 \\
\hline Image 2 & 20.88 & 56.36 & 60.10 & 1.63 & 60.04 & 40.48 & 38.76 & 2225 \\
\hline Image 3 & 21.12 & 54.33 & 61.38 & 2.40 & 61.32 & 40.58 & 36.94 & 1850 \\
\hline Image 4 & 20.58 & 57.00 & 64.03 & 1.66 & 63.91 & 40.39 & 39.91 & 1684 \\
\hline Image 5 & 24.22 & 59.88 & 67.16 & 1.63 & 66.91 & 40.70 & 35.18 & 1585 \\
\hline Image 6 & 32.78 & 52.95 & 61.47 & 1.66 & 61.49 & 40.86 & 35.26 & 1749 \\
\hline Image 7 & 21.31 & 53.29 & 61.13 & 1.70 & 61.15 & 38.95 & 35.73 & 1822 \\
\hline Image 8 & 21.40 & 51.89 & 58.30 & 1.66 & 58.34 & 40.08 & 40.08 & 1990 \\
\hline Image 9 & 20.04 & 50.42 & 59.41 & 1.71 & 59.40 & 38.94 & 36.35 & 2203 \\
\hline
\end{tabular}

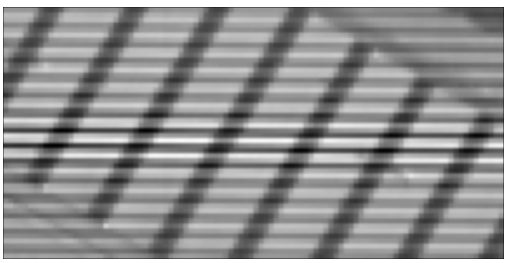

(a)

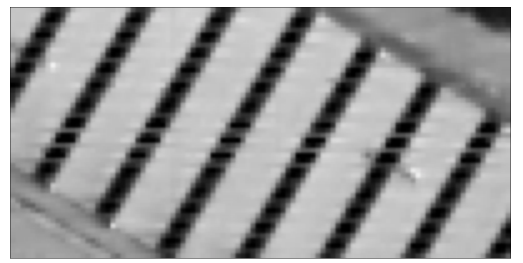

(b)

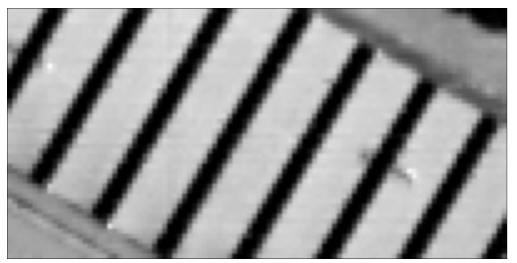

(c)

Fig. 13. Extract of the image shown in Fig. 12. Subimage (a) comes from the measured image. The interference fringes are the horizontal lines. The image in the background corresponds to a building. Subimage (b) comes from the image obtained using the additive scheme with a 2D Fourier transform. Residues of the fringes can be observed. Subimage (c) is the result of the multiplicative algorithm, which successfully removes the residus in (b).

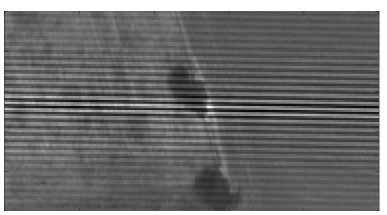

(a)

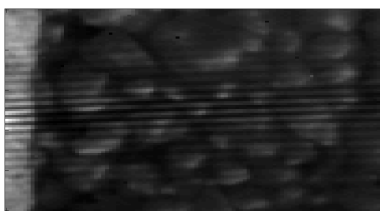

(c)

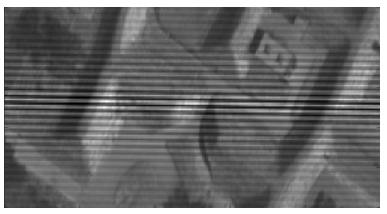

(e)

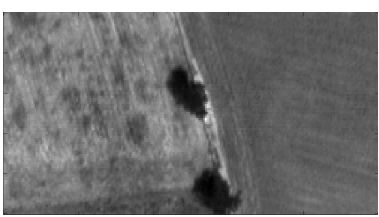

(b)

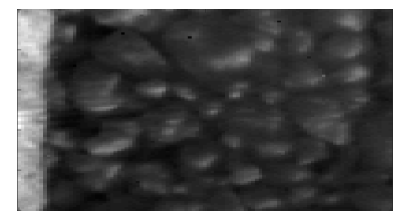

(d)

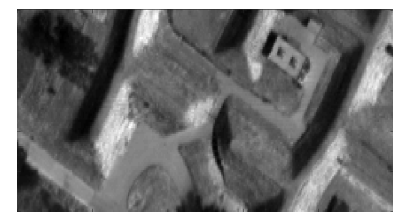

(f)
Fig. 14. Results for various types of landscapes. On the left column, measured images from Sieleters-MWIR, with interference fringes. On the right, the fringes have been removed using Algorithm 3.

non-convex problem of using a mutiplicative image formation model. In turn, such a model allows for a better description of the interferometric image formation process. Furthermore, a good approximation to this solution can be found in a competitive computational time.

The experimental results have shown that the use of smoothed total variation regularization and adopting a 1D

\begin{tabular}{l|c} 
Method & Percent of matched points \\
\hline Additive TV+2DFT & $46 \%$ \\
Measured image & $58 \%$ \\
Multiplicative (Algorithm 2) & $85 \%$
\end{tabular}

TABLE II

STEREOSCOPIC PERFORMANCE FOR THE ADDITIVE MODEL, THE MEASURED IMAGES AND THE MULTIPLICATIVE MODEL.

Fourier transform, well-adapted to the vertical periodicity of interference fringes, considerably improves results. In addition, our 1D regularization scheme retains the well-known spectral extrapolation capability of two-dimensional TV minimization, albeit with some limitations for images with horizontal patterns at the exact same frequency as the fringe pattern.

The use of the smoothed TV was also beneficial for subpixel stereo matching when compared to non-differentiable TV. However, it comes at the price of smoothing out small gradients in the measured image, with an arbitrary contrast threshold. A more accurate (even though more computationally expensive) approach that will be investigated in future research would be to reduce staircasing artifacts by using the non-differentiable Shannon Total Variation proposed by Moisan and Abergel [35], [44].

\section{ACKNOWLEDGEMENT}

This work has been partially funded by the French Research Agency (ANR) under grant No ANR-14-CE27-001 (MIRIAM). 


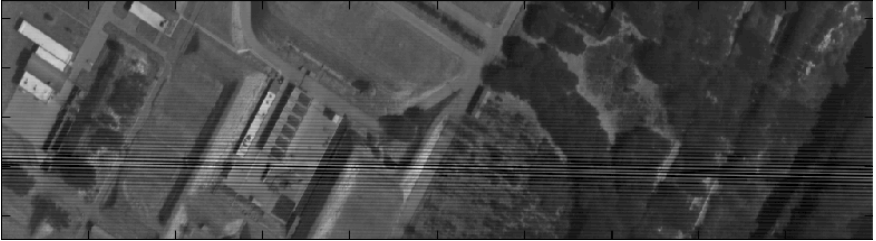

(a) First image: measured

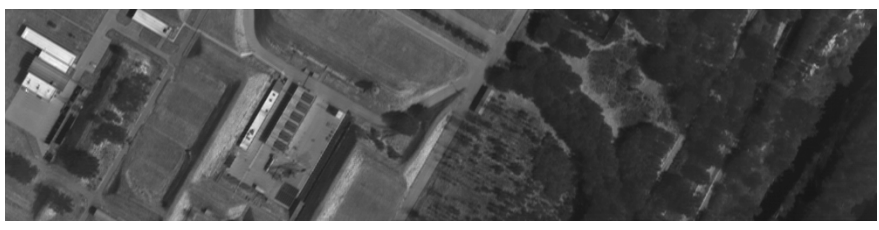

(b) First image: oracle

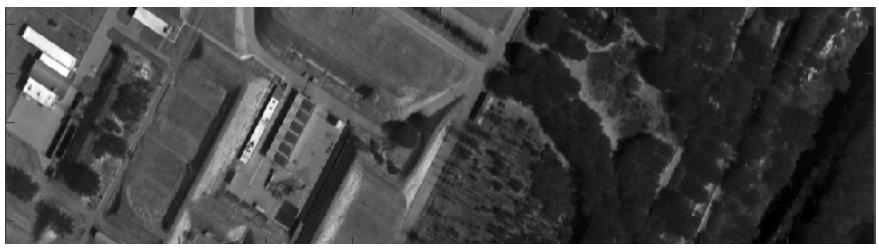

(c) First image: result

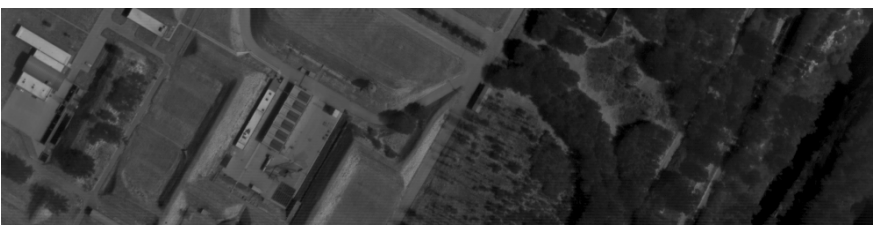

(d) Second image: measured

Fig. 15. Image (a) shows the measured image (from Sieleters-MWIR), (b) the oracle and (c) the image resulting from Algorithm 3 for image (a). (d) is extracted from another image which did not contain contrasted fringes (but light fringes can be observed at the top and bottom of the image) on the same area, for visual comparison.

\section{REFERENCES}

[1] F. D. van der Meer, H. M. A. van der Werff, F. J. A. van Ruitenbeek, C. A. Hecker, W. H. Bakker, M. F. Noomen, M. van der Meijde, E. J. M. Carranza, J. B. d. Smeth, and T. Woldai, "Multi- and hyperspectral geologic remote sensing: A review," International Journal of Applied Earth Observation and Geoinformation, vol. 14, no. 1, pp. 112-128, Feb. 2012. [Online]. Available: http://www.sciencedirect.com/ science/article/pii/S0303243411001103

[2] R. d. R. Salles, C. R. de Souza Filho, T. Cudahy, L. E. Vicente, and L. V. S. Monteiro, "Hyperspectral remote sensing applied to uranium exploration: A case study at the Mary Kathleen metamorphichydrothermal U-REE deposit, NW, Queensland, Australia," Journal of Geochemical Exploration. [Online]. Available: http://www.sciencedirect. com/science/article/pii/S0375674216301455

[3] K. A. Reath and M. S. Ramsey, "Exploration of geothermal systems using hyperspectral thermal infrared remote sensing," Journal of Volcanology and Geothermal Research, vol. 265, pp. 27-38, Sep. 2013. [Online]. Available: http://www.sciencedirect.com/science/article/ pii/S0377027313002412

[4] A. Eisele, S. Chabrillat, C. Hecker, R. Hewson, I. C. Lau, C. Rogass, K. Segl, T. J. Cudahy, T. Udelhoven, P. Hostert, and H. Kaufmann, "Advantages using the thermal infrared (TIR) to detect and quantify semi-arid soil properties," Remote Sensing of Environment, vol. 163, pp. 296-311, Jun. 2015. [Online]. Available: http://www.sciencedirect.com/science/article/pii/S0034425715001236

[5] F. Tavin, A. Roman, S. Mathieu, F. Baret, W. Liu, and P. Gouton, "Comparison of Metrics for the Classification of Soils Under Variable Geometrical Conditions Using Hyperspectral Data," IEEE Geoscience and Remote Sensing Letters, vol. 5, no. 4, pp. 755-759, Oct. 2008. [Online]. Available: http://ieeexplore.iee.org/document/4656457/
[6] C. Spinetti, V. Carrère, M. F. Buongiorno, A. J. Sutton, and T. Elias, "Carbon dioxide of $\mathrm{Pu}^{\prime} \mathrm{u}^{\prime} \mathrm{O}$ 'o volcanic plume at Kilauea retrieved by AVIRIS hyperspectral data," Remote Sensing of Environment, vol. 112, no. 6, pp. 3192-3199, Jun. 2008. [Online]. Available: http://www.sciencedirect.com/science/article/pii/S0034425708001132

[7] H.-h. K. Burke, J. W. Snow, F. W. Chen, and K. E. Farrar, "Satellite hyperspectral IR sensing: investigating water vapor variability using AIRS data," in Proc. SPIE 6233, Algorithms and Technologies for Multispectral, Hyperspectral, and Ultraspectral Imagery XII, vol. 6233, 2006, pp. 62331C-62331C-12. [Online]. Available: http://dx.doi.org/10.1117/12.666572

[8] A. Marino, G. Ludovisi, A. Moccaldi, and S. Bellagamba, "Imaging spectrometry and GIS techniques application for the study of seawater quality and pollution hazard," in Proc. SPIE 4488, Ocean Optics: Remote Sensing and Underwater Imaging, vol. 4488, 2002, pp. 264-268. [Online]. Available: http://dx.doi.org/10.1117/12.452822

[9] A. Fais, P. Nino, and U. Minelli, "Airborne hyperspectral scanner and laser altimeter data application to water reservoirs and water use calculation: first results on two Italian case study," in Proc. SPIE 5976, Remote Sensing for Agriculture, Ecosystems, and Hydrology VII, vol. 5976, 2005, pp. 59760H-59760H-12. [Online]. Available: http://dx.doi.org/10.1117/12.627787

[10] M. Dalponte, L. Bruzzone, and D. Gianelle, "Fusion of Hyperspectral and LIDAR Remote Sensing Data for Classification of Complex Forest Areas," IEEE Transactions on Geoscience and Remote Sensing, vol. 46, no. 5, pp. 1416-1427, May 2008. [Online]. Available: http://ieeexplore.ieee.org/document/4490055/

[11] M. L. Clark and N. E. Kilham, "Mapping of land cover in northern California with simulated hyperspectral satellite imagery," ISPRS Journal of Photogrammetry and Remote Sensing, vol. 119, pp. 228-245, Sep. 2016. [Online]. Available: http://www.sciencedirect.com/ science/article/pii/S0924271616301174

[12] X. Briottet, Y. Boucher, A. Dimmeler, A. Malaplate, A. Cini, M. Diani, H. Bekman, P. Schwering, T. Skauli, I. Kasen, I. Renhorn, L. Klasén, M. Gilmore, and D. Oxford, "Military applications of hyperspectral imagery," in Proc. SPIE 6239, Targets and Backgrounds XII: Characterization and Representation, vol. 6239, May 2006, p. 62390B [Online]. Available: http://adsabs.harvard.edu/abs/2006SPIE.6239E...9B

[13] D. F. Gleeson, R. T. Pappalardo, S. E. Grasby, M. S. Anderson, B. Beauchamp, R. Castaño, S. A. Chien, T. Doggett, L. Mandrake, and K. L. Wagstaff, "Characterization of a sulfur-rich Arctic spring site and field analog to Europa using hyperspectral data," Remote Sensing of Environment, vol. 114, no. 6, pp. 1297-1311, Jun. 2010. [Online]. Available: http://www.sciencedirect.com/science/article/ pii/S0034425710000337

[14] J. Feng, B. Rivard, and A. Sánchez-Azofeifa, "The topographic normalization of hyperspectral data: implications for the selection of spectral end members and lithologic mapping," Remote Sensing of Environment, vol. 85, no. 2, pp. 221-231, May 2003. [Online]. Available: http://www.sciencedirect.com/science/article/pii/S0034425703000026

[15] Z. Yang, C. Yu, W. Zheng, Z. Lei, M. Yan, X. Yuan, and P. Zhang, "Research on ground-based lwir hyperspectral imaging remote gas detection," pp. 96780X-96780X-8, 2015.

[16] A. P. Fossi, Y. Ferrec, N. Roux, O. D'almeida, N. Guerineau, and H. Sauer, "Miniature and cooled hyperspectral camera for outdoor surveillance applications in the mid-infrared," Opt. Lett., vol. 41, no. 9, pp. 1901-1904, May 2016. [Online]. Available: http://ol.osa.org/abstract.cfm?URI=ol-41-9-1901

[17] R. Wright, P. Lucey, S. Crites, H. Garbeil, M. Wood, E. Pilger, A. Gabrieli, and C. Honniball, "Tircis: thermal infrared compact imaging spectrometer for small satellite applications," pp. 98 801K-98 801K-7, 2016. [Online]. Available: http://dx.doi.org/10.1117/12.2224311

[18] F. Wang, J. Zhou, J. Jing, Q. Wu, and W. Cheng, "Research on lasis interferogram processing," pp. 98 110I-98 110I-8, 2015.

[19] C. Coudrain, S. Bernhardt, M. Caes, R. Domel, Y. Ferrec, R. Gouyon, D. Henry, M. Jacquart, A. Kattnig, P. Perrault, L. Poutier, L. RoussetRouvière, M. Tauvy, S. Thétas, and J. Primot, "SIELETERS, an airborne infrared dual-band spectro-imaging system for measurement of scene spectral signatures," Opt Express, vol. 23, no. 12, pp. 16 164-16176, Jun. 2015.

[20] P. G. Lucey, M. Wood, S. T. Crites, and J. Akagi, "A LWIR hyperspectral imager using a sagnac interferometer and cooled $\mathrm{HgCdTe}$ detector array," pp. 83900Q-83900Q-8, 2012. [Online]. Available: http://dx.doi.org/10.1117/12.918970

[21] J. Wen, J. Zhao, and W. Cailing, "Improved MCA-TV algorithm for interference hyperspectral image decomposition," Optics and Lasers 

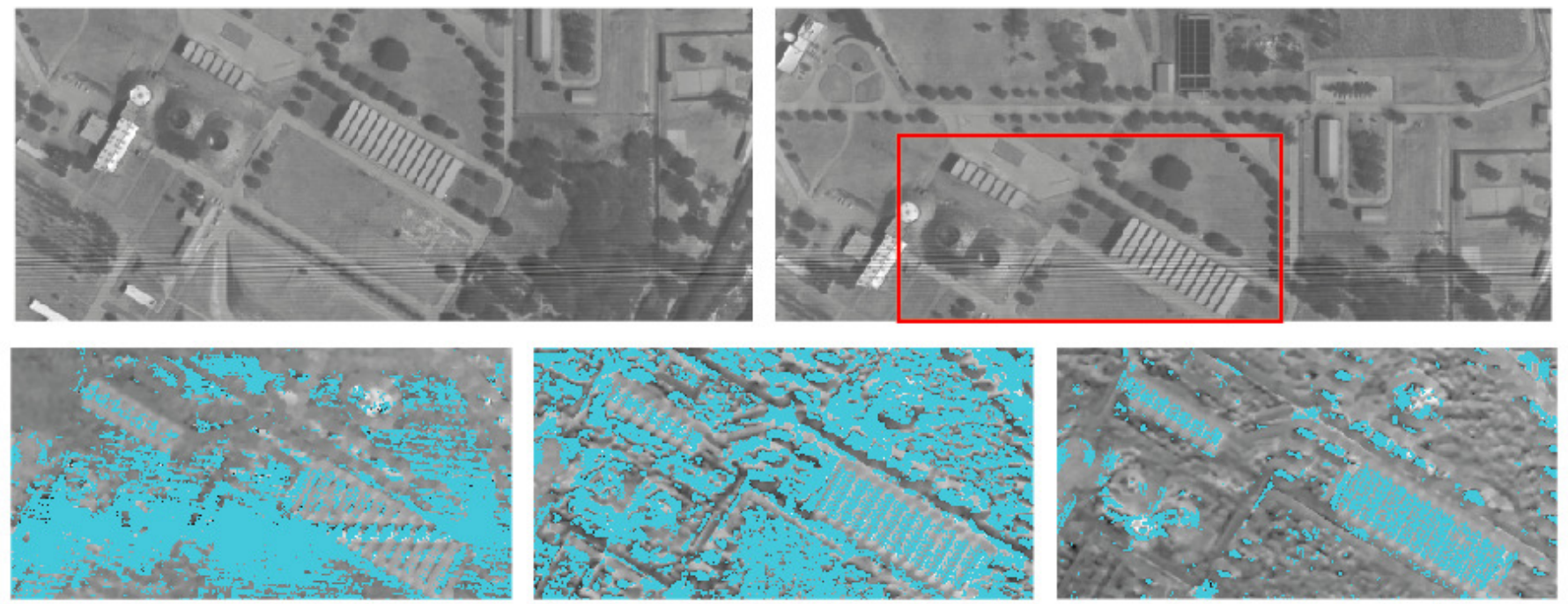

Fig. 16. On the top line, the two images used for stereoscopy, as they were measured. The area designated by a rectangle corresponds to the three disparity maps on the second line. These are obtained, left to right, from measured images, images using the mainstream additive model from section II-B and images using the multiplicative model.

in Engineering, vol. 75, pp. 81-87, Dec. 2015. [Online]. Available: http://linkinghub.elsevier.com/retrieve/pii/S0143816615001530

[22] P. Maurel, J.-F. Aujol, and G. Peyré, "Locally parallel texture modeling," SIAM Journal on Imaging Sciences, vol. 4, no. 1, pp. 413-447, 2011.

[23] X. Zhou, A. G. Podoleanu, Z. Yang, T. Yang, and H. Zhao, "Morphological operation-based bi-dimensional empirical mode decomposition for automatic background removal of fringe patterns," Opt. Express, vol. 20, no. 22, pp. 24247-24262, Oct 2012. [Online]. Available: http://www.opticsexpress.org/abstract.cfm?URI=oe-20-22-24247

[24] Y. Ferrec, J. Taboury, H. Sauer, P. Chavel, P. Fournet, C. Coudrain, J. Deschamps, and J. Primot, "Experimental results from an airborne static fourier transform imaging spectrometer," Appl. Opt., vol. 50, no. 30, pp. 5894-5904, Oct 2011. [Online]. Available: http://ao.osa.org/abstract.cfm?URI=ao-50-30-5894

[25] Y. Ferrec, "Spectro-imagerie aéroportée par transformation de fourier avec un interféromètre statique à décalage latéral : Réalisation et mise en oeuvre," Ph.D. dissertation, Laboratoire Charles Fabry de l'Institut d'Optique Faculté des sciences d'Orsay - Université Pasris-Sud XI, 2008.

[26] S. Osher, A. Solé, and L. Vese, "Image Decomposition and Restoration Using Total Variation Minimization and the $H^{1}$, "Multiscale Modeling \& Simulation, vol. 1, no. 3, pp. 349-370, Jan. 2003. [Online]. Available: http://epubs.siam.org/doi/abs/10.1137/S1540345902416247

[27] J.-F. Aujol, G. Gilboa, T. Chan, and S. Osher, "Structure-texture image decomposition-modeling, algorithms, and parameter selection," International Journal of Computer Vision, vol. 67, no. 1, pp. 111136, 2006. [Online]. Available: http://link.springer.com/article/10.1007/ s11263-006-4331-z

[28] A. Buades, T. M. Le, J. M. Morel, and L. A. Vese, "Fast Cartoon + Texture Image Filters," IEEE Transactions on Image Processing, vol. 19, no. 8, pp. 1978-1986, Aug. 2010.

[29] C. Barbanson, A. Almansa, Y. Ferrec, and P. Monasse, "Correction des effets de relief en spectro-imagerie aéropotée," EDITE, Rapport miparcours, 2016.

[30] L. I. Rudin, S. Osher, and E. Fatemi, "Nonlinear total variation based noise removal algorithms," Physica D: Nonlinear Phenomena, vol. 60, no. 1, pp. 259-268, 1992. [Online]. Available: http: //www.sciencedirect.com/science/article/pii/016727899290242F

[31] F. Malgouyres and F. Guichard, "Edge Direction Preserving Image Zooming: A Mathematical and Numerical Analysis," SIAM J. Numer. Anal., vol. 39, no. 1, pp. 1-37, Jan. 2001. [Online]. Available: http://epubs.siam.org/doi/abs/10.1137/S0036142999362286

[32] A. Almansa, S. Durand, and B. Rougé, "Measuring and Improving Image Resolution by Adaptation of the Reciprocal Cell," Journal of Mathematical Imaging and Vision, vol. 21, no. 3, pp. 235-279, Nov. 2004. [Online]. Available: http://link.springer.com/10.1023/B: JMIV.0000043739.51886.01
[33] C. Barbanson, A. Almansa, Y. Ferrec, and P. Monasse, "Relief Computation from Images of a Fourier Transform Spectrometer for Interferogram Correction," in OSA Light, Energy and Environment Congress - Topical Meeting on Fourier Transform Spectroscopy. Leipzig, Germany: OSA, November 2016, p. FM3E.6. [Online]. Available: https://hal-enpc.archives-ouvertes.fr/hal-01405856

[34] G. Aubert and J.-F. Aujol, "A Variational Approach to Removing Multiplicative Noise," SIAM J. Appl. Math., vol. 68, no. 4, pp. 925946, 2008.

[35] L. Moisan, "How to discretize the Total Variation of an image?" Proc. Appl. Math. Mech., vol. 7, no. 1, pp. $1041907-1041908$, Dec. 2007. [Online]. Available: http://onlinelibrary.wiley.com/doi/10.1002/ pamm.200700424/abstract

[36] G. Aubert and P. Kornprobst, Mathematical problems in image processing. Springer-Verlag Berlin, 2006.

[37] J. Besag, "Digital image processing," Journal of Applied Statistics, vol. 16, no. 3, pp. 395-407, 1989. [Online]. Available: http: //dx.doi.org/10.1080/02664768900000049

[38] M. Nikolova and G. Steidl, "Fast ordering algorithm for exact histogram specification," IEEE Transactions on Image Processing, vol. 23, no. 12, pp. 5274-5283, Dec 2014.

[39] J. Gorski, F. Pfeuffer, and K. Klamroth, "Biconvex sets and optimization with biconvex functions: A survey and extensions," Mathematical Methods of Operations Research, vol. 66, no. 3, pp. 373-407, 2007.

[40] P. L. Combettes and V. R. Wajs, "Signal Recovery by Proximal ForwardBackward Splitting," SIAM MMS, vol. 4, no. 4, pp. 1168-1200, jan 2005.

[41] M. Nikolova and P. Tan, "Alternating proximal forward-backward minimization for nonconvex regularized problems," HAL, 2017.

[42] N. Sabater, A. Almansa, and J.-M. Morel, "Meaningful Matches in Stereovision," IEEE Trans. PAMI, vol. 34, no. 5, pp. 930-42, dec 2011. [Online]. Available: http://hal.archives-ouvertes.fr/hal-00647995

[43] P. Monasse, "Quasi-Euclidean Epipolar Rectification," Image Processing On Line, vol. 1, 2011.

[44] R. Abergel and L. Moisan, "The Shannon Total Variation," 2016. [Online]. Available: https://hal.archives-ouvertes.fr/hal-01349516 


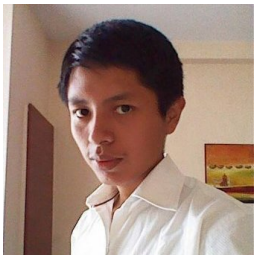

Daniel-Chen Soncco was medalist in various international olympiads in mathematics and informatics. He got his bachelor degree in Mathematics at Universidad Nacional de Ingeniería (Lima, Peru) in 2012 and received his master's degree from Ecole Polytechnique (France, promotion X2012) in 2016. He conducted his master's thesis on image processing and inverse problems at CMLA, CNRS and ENS Paris-Saclay, France. Currently he is working at Amadeus in Sophia Antipolis as a Junior Programmer.

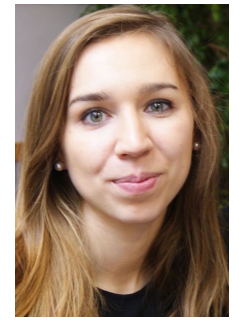

Clara Barbanson received her master's degree from Ecole Supérieure des Géomètres et Topographes (France) in 2014. Her graduation thesis was carried on at the French Mapping Agency on land cover classification using hyperspectral and lidar data. She is currently a Ph.D. candidate at the French Aerospace Lab (ONERA) and Images group in the Signal and Image Processing department at Telecom Paristech (France). Her research interests are inverse problems and stereoscopy.

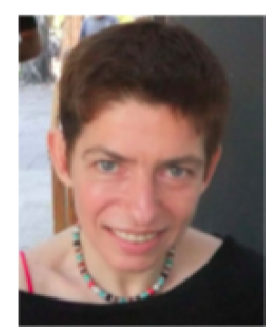

Mila Nikolova received a Ph.D. degree in 1995 and a Habilitation degree in 2006. Currently, she is Research Director with the National Center for Scientific Research (CNRS), France. She performs her duty as a full-time researcher at CMLA (Centre de Mathématiques et de Leurs Applications), CNRS and ENS Cachan, France. Her research interests are in mathematical image and signal reconstruction, Inverse problems, regularisation and variational methods and the properties of their solutions, scientific computing.

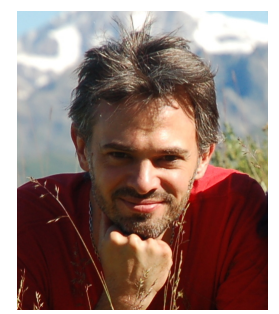

Andres Almansa received his HDR (2005), Ph.D. (2002) and M.Sc.(1998)/Engineering(1995) degrees in Applied Mathematics and Computer Science from Université Paris-Descartes, ENS Cachan (France) and Universidad de la Republica (Uruguay), respectively, where he served as an Associate Professor for several years. Since 2007 he works with CNRS, first as a Research Scientist at LTCI - Telecom ParisTech, and later (since 2016) as a Research Director at MAP5 - Université Paris Descartes. His current interests include image restoration and analysis, subpixel stereovision and applications to earth observation, high quality digital photography and film restoration.

Yann Ferrec received his Ph.D. degree in 2008 from Université Paris-Sud XI (France). He is currently a researcher at the French Aerospace Lab (ONERA) in France. His research interests are spectrometry and hyperspectral imaging systems. 